\title{
Therapeutic targeting of endoplasmic reticulum stress in acute graft-versus-host disease
}

Eileen Haring, ${ }^{1,2,3}$ Geoffroy Andrieux, ${ }^{3,4}$ Franziska M. Uhl, ${ }^{1,2}$ Máté Krausz, ${ }^{5}$ Michele Proietti, ${ }^{5}$ Barbara Sauer, ${ }^{1}$ Philipp R. Esser, ${ }^{6}$ Stefan F. Martin, ${ }^{6}$ Dietmar Pfeifer, ${ }^{1}$ Annette Schmitt-Graeff, Justus Duyster, ${ }^{1}$ Natalie Köhler,,8 Bodo Grimbacher, $, 5,8,9,10$ Melanie Boerries, ${ }^{3,4,11}$ Konrad Aumann, ${ }^{12}$ Robert Zeiser ${ }^{1,4,8}$ and Petya Apostolova ${ }^{1,3}$

1'Department of Medicine I, Medical Center - Faculty of Medicine, University of Freiburg, Freiburg; ${ }^{2}$ Faculty of Biology, Albert-Ludwigs-University, Freiburg; ${ }^{3}$ German Cancer Consortium (DKTK), Partner site Freiburg and German Cancer Research Center (DKFZ), Heidelberg; ${ }^{4}$ Institute of Medical Bioinformatics and Systems Medicine, Medical Center - Faculty of Medicine, University of Freiburg, Freiburg; ${ }^{5}$ Institute for Immunodeficiency, Center for Chronic Immunodeficiency (CCl), Medical Center, Faculty of Medicine, Albert-Ludwigs-University, Freiburg; ${ }^{6}$ Allergy Research Group, Department of Dermatology, Medical Center - Faculty of Medicine, University of Freiburg, Freiburg; ${ }^{7}$ University of Freiburg, Freiburg; ${ }^{8} \mathrm{CIBSS}-$ Center for Integrative Biological Signalling Studies, University of Freiburg, Freiburg; ${ }^{9}$ DZIF - German Center for Infection Research, Satellite Center Freiburg, Freiburg; ${ }^{10}$ RESIST - Cluster of Excellence 2155 to Hannover Medical School, Satellite Center Freiburg, Freiburg;

${ }^{11}$ Comprehensive Cancer Center Freiburg (CCCF), Medical Center - Faculty of Medicine, University of Freiburg, Freiburg, and ${ }^{12}$ Department of Pathology, Medical Center - University of Freiburg, Faculty of Medicine, University of Freiburg, Freiburg, Germany

\author{
Correspondence: \\ Petya Apostolova \\ petya.apostolova@uniklinik-freiburg.de \\ Received: January 17, 2021. \\ Accepted: $\quad$ August 6, 2021. \\ Prepublished: $\quad$ August 19, 2021. \\ https://doi.org/10.3324/haematol.2021.278387 \\ @2022 Ferrata Storti Foundation \\ Haematologica material is published under a CC \\ BY-NC license @() $\Theta$
}

\begin{abstract}
Acute graft-versus-host disease (GvHD) is a life-threatening complication of allogeneic hematopoietic cell transplantation (allo-HCT), a potentially curative treatment for leukemia. Endoplasmic reticulum (ER) stress occurs when the protein folding capacity of the ER is oversaturated. How ER stress modulates tissue homeostasis in the context of alloimmunity is not well understood. We show that ER stress contributes to intestinal tissue injury during GVHD and can be targeted pharmacologically. We observed high levels of ER stress upon GvHD onset in a murine alloHCT model and in human biopsies. These levels correlated with GVHD severity, underscoring a novel therapeutic potential. Elevated ER stress resulted in increased cell death of intestinal organoids. In a conditional knockout model, deletion of the ER stress regulator transcription factor Xbp1 in intestinal epithelial cells induced a general ER stress signaling disruption and aggravated GVHD lethality. This phenotype was mediated by changes in the production of antimicrobial peptides and the microbiome composition as well as activation of pro-apoptotic signaling. Inhibition of inositol-requiring enzyme $1 \alpha$ (IRE1 $\alpha$ ), the most conserved signaling branch in ER stress, reduced GvHD development in mice. IRE1 $\alpha$ blockade by the small molecule inhibitor $4 \mu 8 \mathrm{c}$ improved intestinal cell viability, without impairing hematopoietic regeneration and T-cell activity against tumor cells. Our findings in patient samples and mice indicate that excessive ER stress propagates tissue injury during GVHD. Reducing ER stress could improve the outcome of patients suffering from GvHD.
\end{abstract}

\section{Introduction}

Acute graft-versus-host disease (GvHD) is a life-threatening complication of allogeneic hematopoietic cell transplantation (allo-HCT). In particular, GVHD of the gastrointestinal tract (GI-GVHD) remains one of the most frequent causes of allo-HCT-related morbidity and mortality. ${ }^{1}$ GI-GVHD results from a complex multi-step crosstalk between extensive epithelial tissue damage in the patient and activation of the allo-reactive immune system transferred with the donor graft. ${ }^{2}$ Enterocytes are subjected to cellular stress and undergo apoptosis. As a result, patients can develop diarrhea, dehydration, intestinal bleeding, hypalbuminemia, and generalized infections. ${ }^{2}$ Recently, it has been shown that GVHD is also associated with changes of the microbiome diversity and composition. ${ }^{3}$ Standard treatment for GI-GVHD consists of corticosteroids or other compounds that target immune cell activation. ${ }^{4}$ Several factors supporting intestinal repair mechanisms have been identified in pre-clinical studies, 
including IL-22,, ${ }^{5,6}$ R-spondin ${ }^{7,8}$ and glucagon-like peptide 2. ${ }^{9}$ Recently, we have demonstrated a protective role for bile acids, which reduce cytokine-mediated intestinal injury and decrease intestinal antigen presentation. ${ }^{10}$ These novel developments show as a proof-of-principle that local regulation of repair mechanisms and inflammation is a successful approach to treat GI-GVHD and possibly other inflammatory diseases of the intestine.

In this study, we set out to decipher the role of endoplasmic reticulum (ER) stress, a cellular stress reaction, in the context of GI-GvHD. ER stress occurs upon accumulation of unfolded proteins in the ER lumen which can result from hypoxia, tissue damage, or pathogen exposure. These unfolded proteins are sensed by three molecules in the ER membrane which activate signaling cascades, commonly known as the unfolded protein response (UPR). ${ }^{11}$ The most conserved UPR transducer is the kinase inositol-requiring enzyme $1 \alpha$ (IRE1 $\alpha$ ). This kinase has a ribonuclease activity and splices the mRNA of the transcription factor $X$-box binding protein 1 (XBP1), which then translocates to the nucleus and regulates the expression of chaperones, foldases and enzymes for lipid metabolism..$^{12,3}$ The two other UPR branches are mediated by the protein kinase RNA-like ER kinase (PERK) and the activating transcription factor 6 (ATF6)..$^{11}$ Their downstream signaling cascades lead to a reduction of the intracellular protein overload. The fundamental task of the UPR is to assist the cell in coping with the large amount of unfolded proteins. However, prolonged activation of the UPR also activates pro-apoptotic kinases and transcription factors. ${ }^{11,14,15}$ Another mechanism contributing to ER stress-induced cell death is mediated via regulated inositol-requiring enzyme 1-dependent decay of mRNA (RIDD). ${ }^{16}$ During this process, IRE $1 \alpha$ splices and thus inactivates multiple mRNA which results in cell death. These opposite effects of ER stress on cell fate underline the fact that a tight regulation of the UPR is necessary to maintain physiological balance and homeostasis in stressed cells (Figure 1A).

Our study aimed to close the gap in understanding the function of intestinal UPR and its therapeutic potential in GI-GVHD. We observed that ER stress occurred during GVHD development in mice and humans and correlated with GI-GVHD onset and severity. Using intestinal organoids and a genetic conditional knockout mouse model, we found that dysregulated chronic ER stress reduced intestinal cell viability and aggravated GvHD severity by altercating anti-microbial peptide production, the microbiome composition and pro-apoptotic pathway activity. Pharmacological inhibition of IRE1 $\alpha$, one of three ER stress signaling pathways, improved GVHD outcome by directly protecting the intestinal epithelium. Importantly, this intervention did not impair hematopoietic regeneration, T-cell proliferation or anti-tumor killing capacity.
Our study proposes the inhibition of this ER stress pathway as a novel approach for the treatment of GI-GvHD.

\section{Methods}

\section{Mice}

BALB/C $\left(\mathrm{H}-2 \mathrm{~K}^{\mathrm{d}}\right)$ and C57BL/6 $\left(\mathrm{H}-2 \mathrm{~K}^{\mathrm{b}}\right)$ mice were purchased from Janvier Labs (Le Genest-Saint-Isle, France). Luciferase-transgenic C57BL/6 mice $\left(\mathrm{H}-2 \mathrm{~K}^{\mathrm{b}}\right)$ were bred in the animal facility of the Center for Clinical Research at the Medical Center - University of Freiburg, Germany. Mice carrying a floxed $X b p 1$ allele $\left(X b p 1^{f l / f l}\right)$ have been previously described ${ }^{17}$ and were a kind gift from Dr. Laurie Glimcher, Cornell University, USA. B6. Cg-Tg(Vil'-cre $) 997 \mathrm{Gum} / \mathrm{J}$ mice expressing the Cre recombinase in villus and crypt intestinal epithelial cells (Vilcre) were purchased from the Jackson Laboratory. VilcreXbp $7^{\mathrm{fl} / \mathrm{fl}}$ mice and $X b p 7^{\mathrm{fl} / \mathrm{fl}}$ littermates were generated by crossing these two strains. All animals were housed under specific pathogen-free conditions at the animal facility of the Center for Clinical Research (ZKF, Freiburg, Germany). Genotypes were confirmed via polymerase chain reaction (PCR). All animal protocols (G16/018, G-17/063, G-18/063; X-13/07J; X-15/10A; X20/06K) were approved by the Federal Ministry for Nature, Environment and Consumer Protection of the state of BadenWürttemberg, Germany.

All other methods are provided in the Online Supplementary Appendix.

\section{Results}

\section{Unfolded protein response is activated during early graft-versus-host disease development in mice}

We first characterized the UPR activity in the intestine of healthy mice and after GVHD induction by quantifying the splicing of $X b p 1$, which is one of the initiating events of UPR signaling. We observed increased Xbp1 splicing in the colon of mice 7 days after allo-HCT when compared to untreated mice (Online Supplementary Figure S1A-C). In order to study the exact time course of UPR activation, we performed quantification of the established ER stress markers Xbp1s/Xbp1u (spliced Xbp1 in relation to unspliced Xbp1) and Hspa5 (encoding for the chaperone GRP78, also known as BIP) at multiple time points after allo-HCT (Figure 1B). We further set out to distinguish whether ER stress was induced as a consequence of the conditioning treatment alone (total body irradiation [TBI]) or by the allo-immune reaction that marks GvHD development. We observed an upregulation of the UPR-related markers in the small intestine (Online Supplementary Figure S1D) and in the colon (Figure 1C) of both TBI and GVHD mice when compared to healthy mice. While ER stress markers were 
A

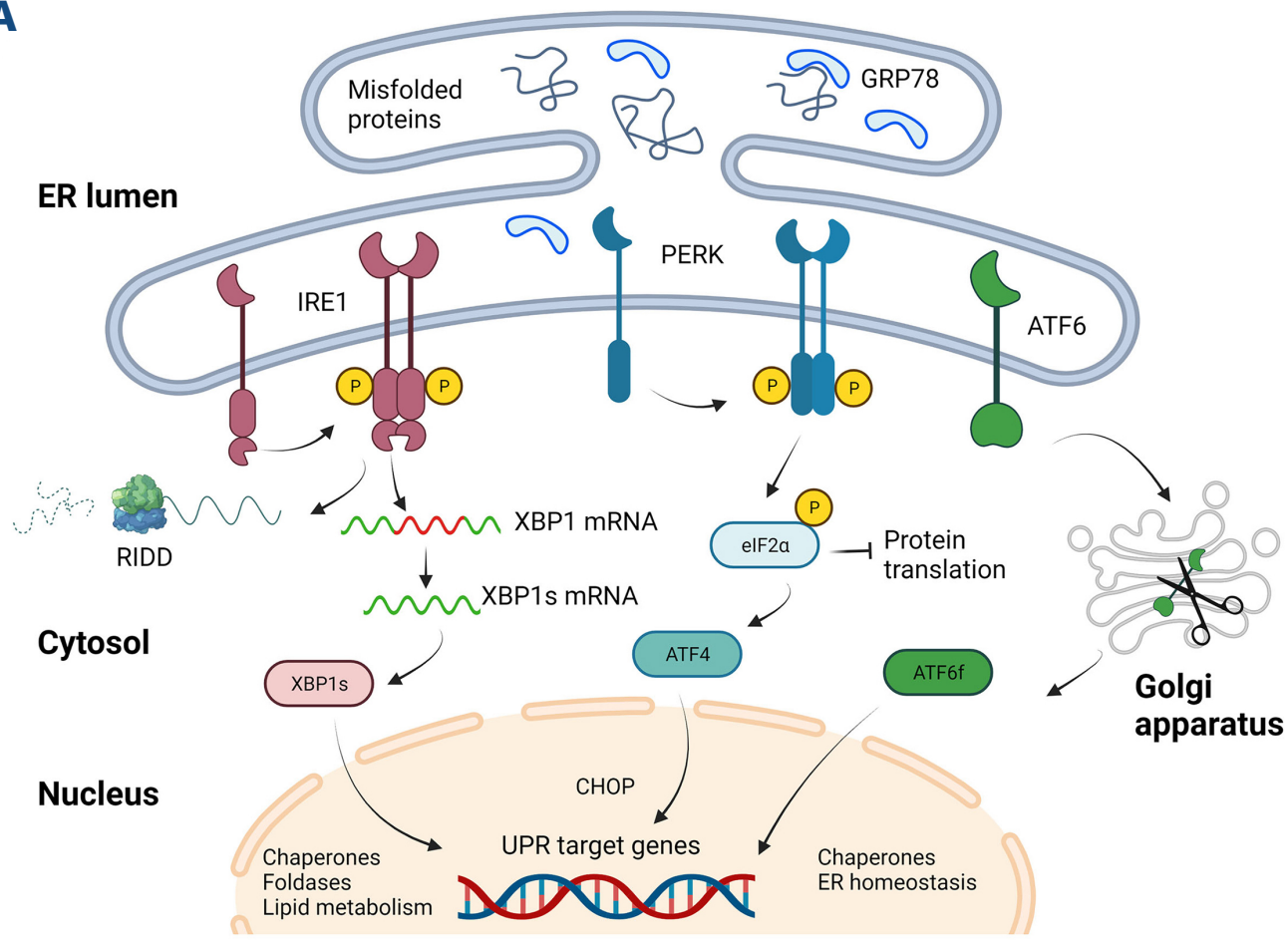

B
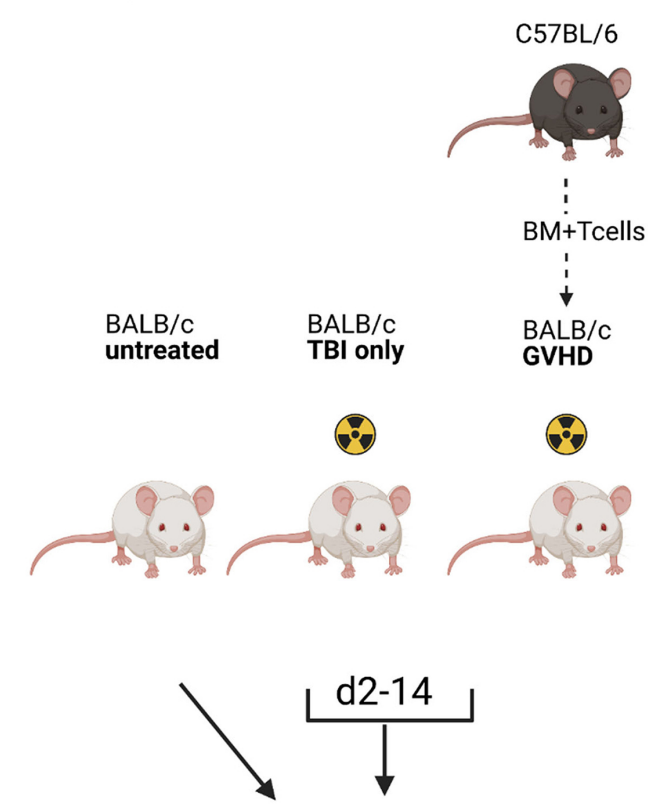

Isolation of the intestine
C

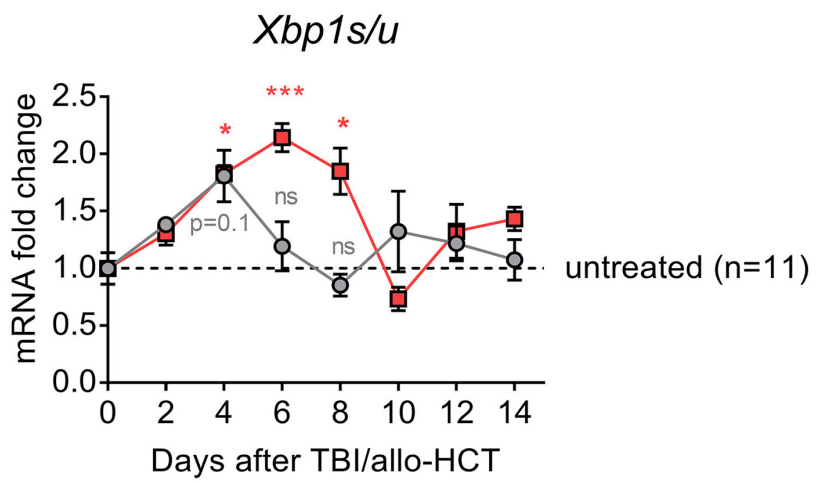

D

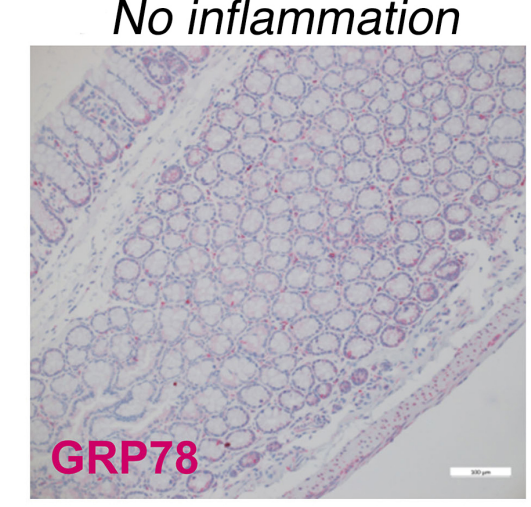

$\mathbf{F}$
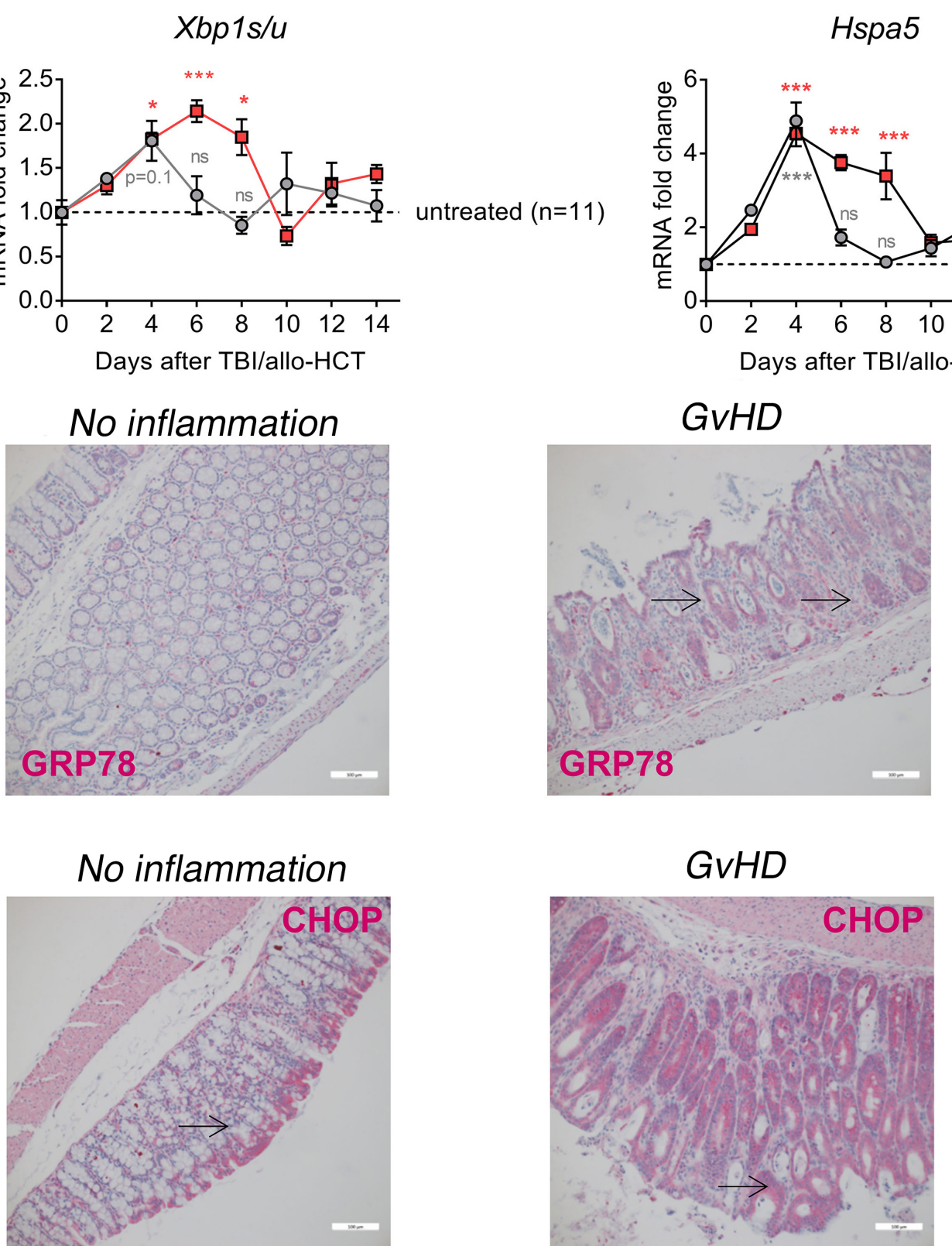

-. $T B I(n=5-7)$

ㅁ. GVHD $(n=6-7)$

untreated $(n=11)$

E

IHC score

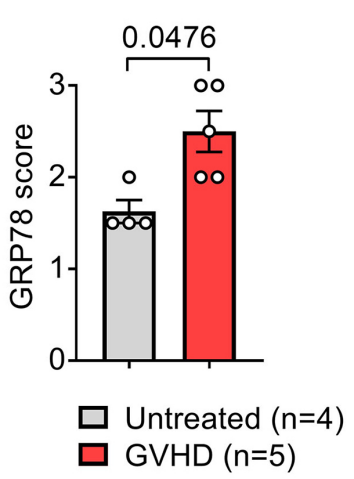

G

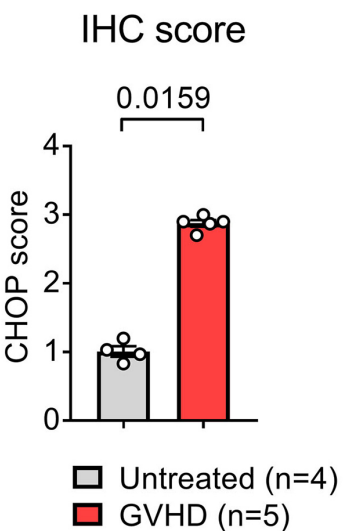

Figure 1. Graft-versus-host disease induction leads to endoplasmic reticulum stress in the murine intestine. (A) Schematic overview of the three different unfolded protein response (UPR) branches induced by endoplasmic reticulum (ER) stress, created with BioRender.com. (B, C) BALB/c mice received either only total body irradiation (TBI) with 10 Gy or underwent complete allogeneic hematopoietic cell transplantation (allo-HCT) as described in Methods with $5 \times 10^{6}$ bone marrow (BM) cells and $3 \times 10^{5}$ $\mathrm{CD}^{+}$and $\mathrm{CD} 8^{+} \mathrm{T}$ cells isolated from the spleen of a C57BL/6 donor. Untreated BALB/c mice were used as a control. (B) Overview of the experimental setup, created with BioRender.com. (C) Quantitative real-time polymerase chain reaction (PCR) analysis of the mRNA expression of selected UPR marker genes in the colon with Actb as a reference gene. Samples were isolated on different time points after TBI or allo-HCT as indicated. The dashed line represents the expression level in untreated mice, which 
was set to 1. Data were pooled from $n=11$ mice for day $0(\mathrm{~d} 0)$ and $n=5-7$ mice/group for the other time points. The $P$-values were calculated using the ordinary one-way ANOVA with correction for multiple comparisons. Statistical comparisons between "GVHD" and "untreated" are highlighted in red color, statistical comparisons between "TBI" and "untreated" are highlighted in grey color, ${ }^{*} P<0.05, * \star * P<0.0001$. (D) Representative immunohistochemistry staining images for GRP78 in murine colon tissue sections. Sections from an untreated mouse ("no inflammation") and from an allo-transplanted BALB/c mouse ("GvHD") on d7 after allo-HCT are shown. Arrows point to GRP78-expressing cells. Scale bars $100 \mu \mathrm{M}$. (E) Quantification of the GRP78 expression score. Each dot represents a single mouse, $n=4-5$ mice/group. The $P$-value was calculated using a two-tailed unpaired Mann Whitney $U$ test. (F) Representative immunohistochemistry staining images for CHOP in murine colon tissue sections. Sections from an untreated mouse ("no inflammation") and from an allo-transplanted BALB/c mouse ("GvHD") on d7 after allo-HCT are shown. Arrows point to GRP78-expressing cells. Scale bars $100 \mu \mathrm{M}$. (G) Quantification of the CHOP expression score. Each dot represents a single mouse, $n=4-5$ mice/group. The $P$-value was calculated using a two-tailed unpaired Mann Whitney $U$ test.

induced by TBI as early as day 4, they dropped back to normal more rapidly than in mice with GVHD. Especially 6-8 days after GVHD induction in our model, Xbp1s/Xbp1u and Hspa5 expression were significantly higher in mice developing GVHD compared to TBI mice and healthy controls (Figure 1C). After that time point, ER stress levels in the colon declined and were similar to baseline (Figure 1C) whereas in the small intestine a second increase was observed on day 14 after allo-HCT (Online Supplementary Figure S1D). These data indicate an active UPR during early GI-GvHD development. While we observed that TBI alone can induce the UPR, transfer of T cells aggravated ER stress additionally. In line with this, immunohistochemistry for GRP78 showed increased expression in the colon of GVHD mice when compared to healthy colon tissue (Figure 1D to E). Although the aim of the UPR is to support the cellular survival in stress conditions by reducing the load of unfolded proteins, pro-apoptotic cascades are engaged during prolonged ER stress. C/EBP homologous protein (CHOP, encoded by the gene Ddit3) is one of the transcription factors mediating apoptosis in the context of the UPR. We found that CHOP is expressed by the healthy colonic epithelium, especially at the top of the crypts. Interestingly, the expression in GvHD-developing mice extended to the whole crypt (Figure 1F, G). As epithelial regeneration has its origin at the bottom of the crypts where intestinal stem cells reside, CHOP-induced apoptosis in this area might reduce the capacity of the intestine to regenerate itself. Together, we observed that GI-GVHD development in mice is marked by increased levels of ER stress.

\section{Unfolded protein response is activated during graft-versus-host disease (GvHD) onset in humans and correlates with GvHD severity}

GI-GVHD is a major factor contributing to morbidity and mortality in allo-HCT recipients. In order to assess whether the UPR was activated in humans, we examined biopsies of GVHD patients for the expression of GRP78 and CHOP. Healthy tissue and biopsies from colitis patients were used as negative and positive controls, respectively. In the uninflamed intestine, expression of GRP78 was confined to immune cells that were interspersed between crypts, and staining in epithelial cells was low (Figure 2A). Confirming a previously shown connection between ER stress and colitis, ${ }^{17}$ GRP78 expression in the epithelium was strong in colitis samples. When analyzing biopsies from GvHD patients, we observed increased expression in higher-grade GvHD compared to lower-grade GVHD (Figure $2 \mathrm{~A}$ and B). Similarly, healthy colon tissue showed only low expression of $\mathrm{CHOP}$. We observed a more intensive $\mathrm{CHOP}$ signal in biopsies from patients with GVHD grade 2-3, especially from injured crypts that were undergoing cell death (Figure 2C). Histology data were supported by the analysis of a publicly available RNA sequencing data set (GSE134662), generated by Holtan et al.18 We found an upregulation of various ER stress-related genes when comparing gut biopsies from newly diagnosed or steroid-refractory GvHD to healthy tissue (Online Supplementary Figure S2A, B). Gene set enrichment analysis confirmed a differential expression of genes matched to the GO term "Response to endoplasmic reticulum stress" (Online Supplementary Figure S2C). Collectively, our data show that ER stress occurs during GVHD development in mice and humans, and that its levels correlate with GVHD severity.

\section{Chronic endoplasmic reticulum stress aggravates intestinal cell death and graft-versus-host disease}

Based on our observation that the UPR is activated during GvHD, we investigated the cellular impact of ER stress induction on the intestine using murine small intestinal organoids that were established and cultured as previously described..$^{19}$ Exposure of organoids to the ER stress inducer tunicamycin resulted in cell death as observed by microscopy and flow cytometry (Figure 3A-D). Release of DAMP from dying cells contributes to the activation of antigenpresenting cells in the context of GVHD. ${ }^{20,21}$ We detected increased concentrations of the DAMP uric acid in the supernatants of tunicamycin-treated organoids (Figure $3 E$ ). Next, we investigated if UPR pathway disruption affects GVHD outcome in a murine allo-HCT model. Since the IRE1 $\alpha$ pathway is the most conserved branch among different species, we focused on the key transcription factor XBP1 which is downstream of IRE1 $\alpha$ and gets activated by the RNase activity of this enzyme. In order to test whether 
intestinal Xbp1 plays a role for the development of GvHD, we crossed $\mathrm{Vil}$ (cre mice bearing the Cre-recombinase under the control of the Villin-promoter with $X b p 1^{f l / f l}$ mice carrying a floxed exon 2 in the $X b p 1$ gene. The specific deletion of the exon 2 of $X b p 1$ in the intestine of $V_{i l} l^{\mathrm{Cre}} X b p 7^{\mathrm{fl} / \mathrm{fl}}$ mice (here referred to as $X b{ }^{\triangle \triangle E C}$ ) was confirmed by quantitative PCR analysis of the intestine in comparison to $X b p 1^{f l / f l}$ (here referred to as $X b p 1^{+/+}$) littermate controls (Online Supplementary Figure $S 3 A$ ). Subsequently, we asked if intestinal $X b p 1$ deletion had an impact on the development of GVHD. Survival of Xbp $1^{1 \mathrm{EEC}}$ mice after induction of GVHD was significantly reduced when compared to $\mathrm{Xbp1}^{+/+}$littermate controls (Figure 3F, G). Furthermore, his- topathological GvHD scores were increased in $X b p 1^{\triangle I E C}$ mice in two different allo-HCT models with TBI or chemotherapy as a conditioning treatment (Figure $3 \mathrm{H}$; Online Supplementary Figure S3B, C). Altogether, these data show that chronic ER stress induces intestinal cell death and results in deteriorated GVHD outcome in different murine models.

\section{Intestinal deletion of Xbp1 alters anti-microbial peptides and microbiome composition of the intestine}

Next, we explored the mechanism by which chronic ER stress increased GvHD. Gene expression analysis revealed that multiple genes belonging to the defensin (Def) family
A
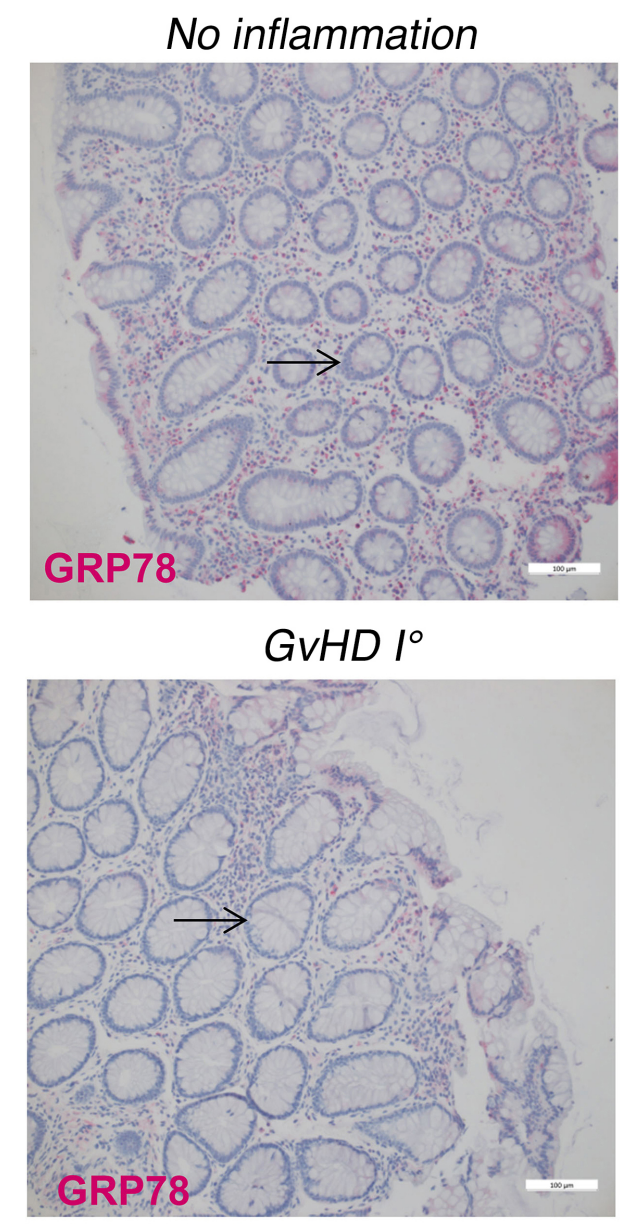

C

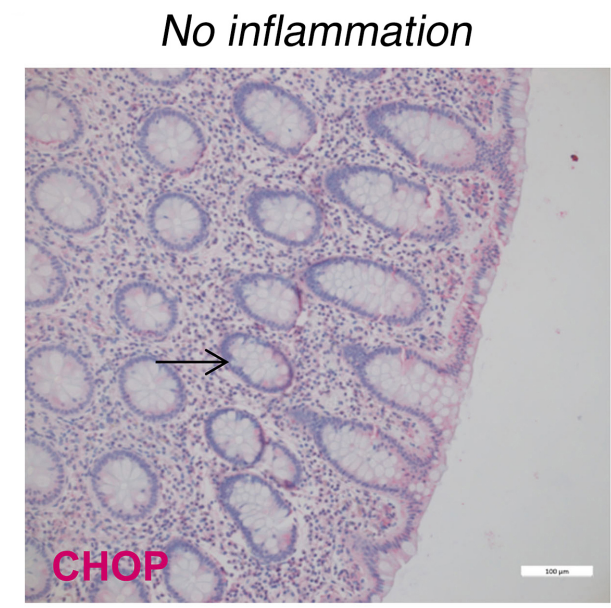

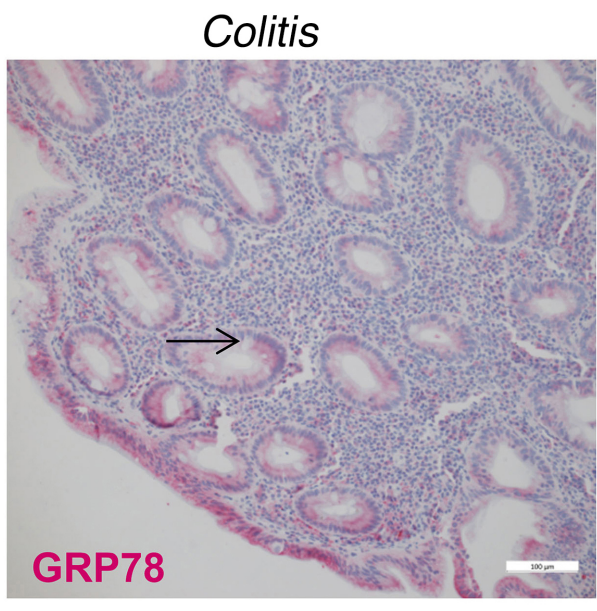

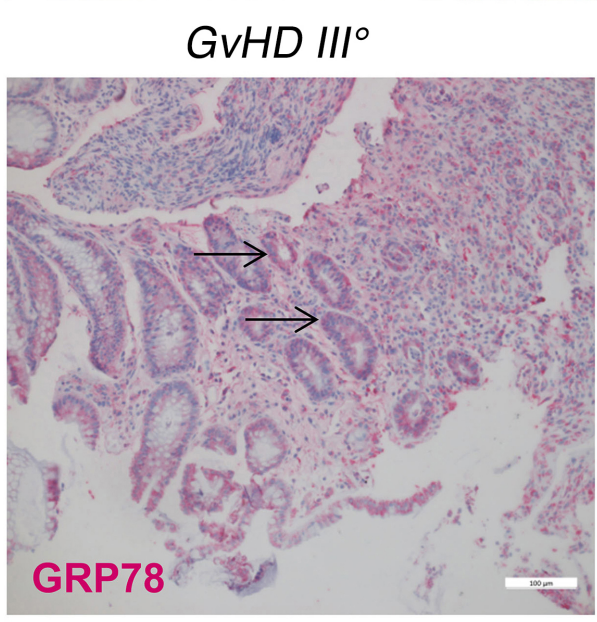

B

IHC score

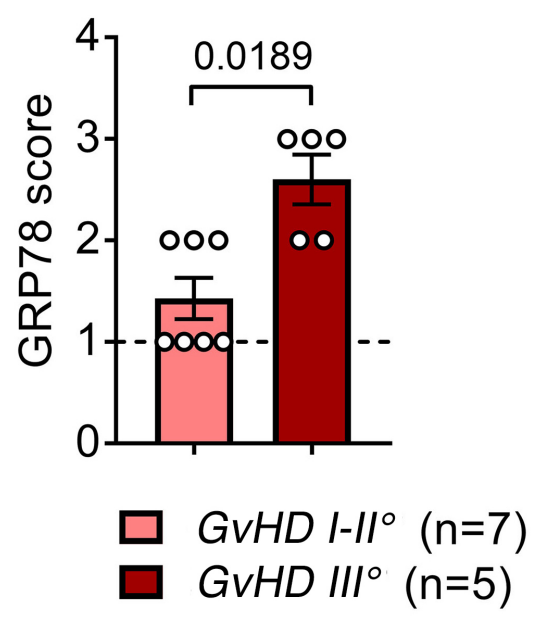

Figure 2. Levels of endoplasmic reticulum stress markers correlate with graft-versus-host disease severity in patients. (A, B) Immunohistochemistry staining for GRP78 in human colon tissue samples. (A) Representative images from healthy and diseased colon tissues as indicated. Arrows point to GRP78-expressing cells. The colitis sample served as a positive control. Scale bars $100 \mu \mathrm{m}$. (B) Quantification of the GRP78 expression score in graft-versus-host disease (GvHD) samples. Statistical analysis of n=7 (GvHD grade 1-2) and $n=5$ (GvHD grade 3) patient biopsies. The $P$-value was calculated using the two-tailed unpaired Mann Whitney $U$ test. (C) Representative images of an immunohistochemistry staining for CHOP in human healthy and diseased colon tissues as indicated. Arrows point to CHOP-expressing cells. Scale bars $100 \mu \mathrm{m}$. 
A

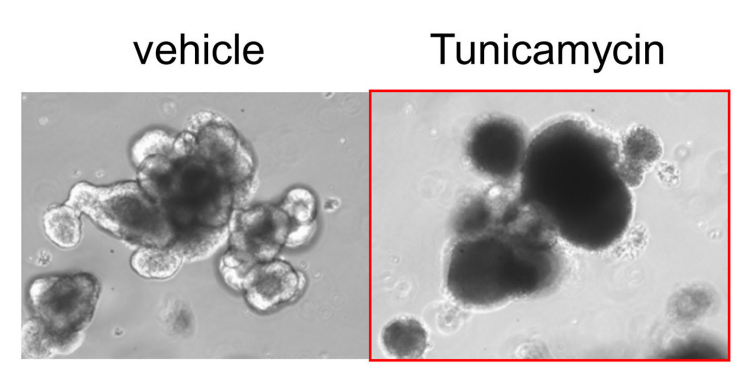

B

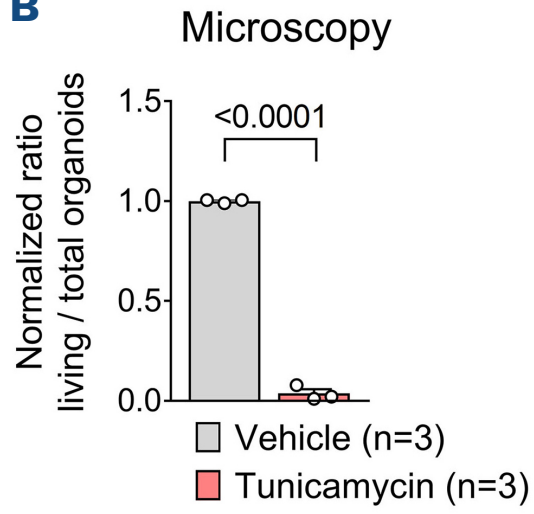

C

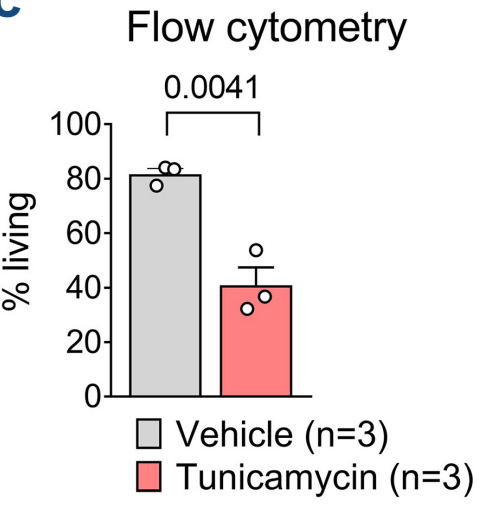

D

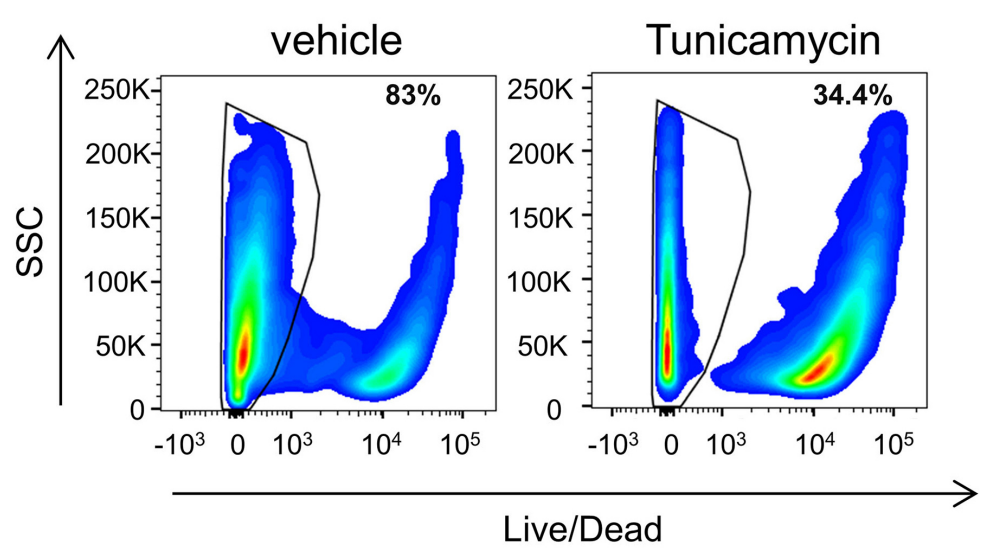

E

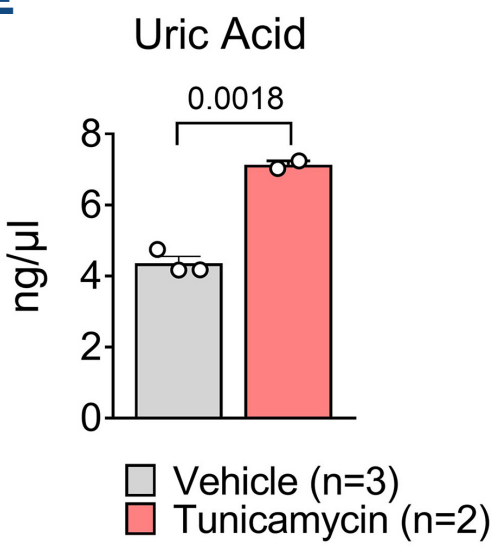

$\mathbf{F}$

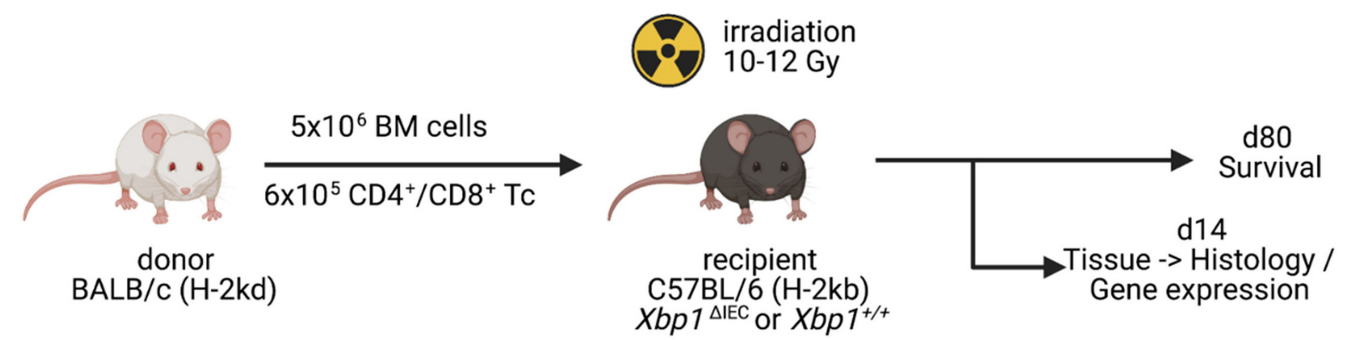

G

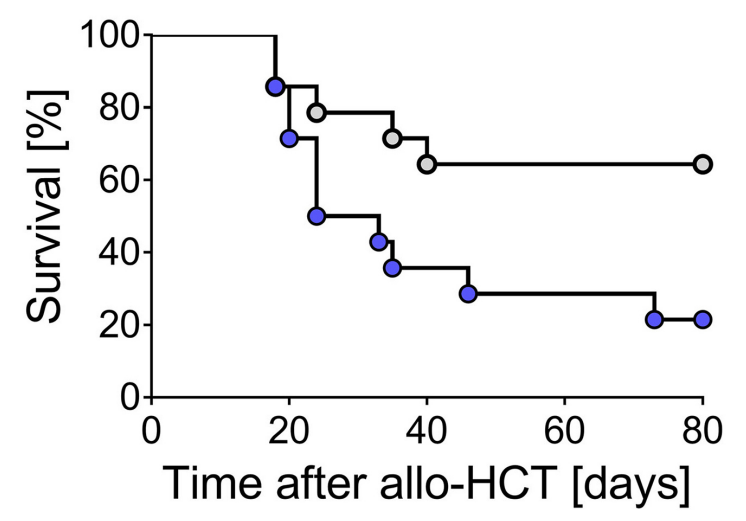

H
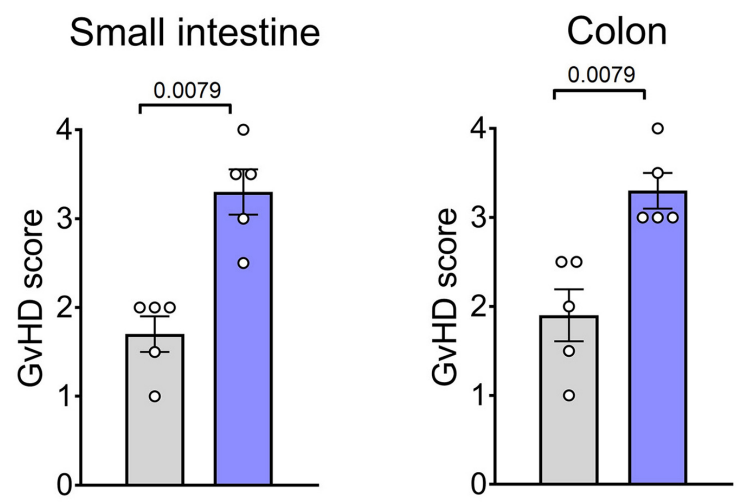

$$
\left.\begin{array}{l}
-X b p 1^{+/+} \text {recipient }(\mathrm{n}=14) \\
-\mathrm{Xbp}^{\mathrm{\Delta IEC}} \text { recipient }(\mathrm{n}=14)
\end{array}\right] 0.0299
$$

$\square \times b p 1^{+/+}$recipient $(\mathrm{n}=5)$

$\square \times b p 1^{\Delta \mathrm{IEC}}$ recipient $(\mathrm{n}=5)$

Figure 3. Chronic endoplasmic reticulum stress induces intestinal cell death and a more severe graft-versus-host disease phenotype. (A) Representative images of BALB/c intestinal organoids treated for 24 hours (h) with $1 \mu \mathrm{g} / \mathrm{mL}$ tunicamycin or vehicle control. (B) Quantification of living organoids after vehicle or tunicamycin treatment as in (A) performed by manual microscope counting. Data were normalized to the vehicle group. Statistical analysis of $\mathrm{n}=3$ biologically independent experiments. The $P$ value was calculated using the two-tailed unpaired Student's $t$-test. (C, D) Intestinal organoids were cultured as described in (A). Organoids were digested and the proportion of dead cells was determined by flow cytometry. (C) Quantification of the percentage of dead cells. The $P$-value was calculated using the two-tailed unpaired Student's $t$-test. (D) Representative flow cytometry dot plots. (E) Organoids were treated with $0.15 \mu \mathrm{g} / \mathrm{mL}$ tunicamycin for $12 \mathrm{~h}$ and afterwards allowed to rest for further $24 \mathrm{~h}$ in normal medium. Levels of uric acid were measured in the supernatant medium, in which the organoids were rested. Concentrations were measured in total $\mathrm{ng} / \mu \mathrm{L}, \mathrm{n}=2-3$ individual biological replicates/group. The $P$-value was calculated using the two-tailed unpaired Student's $t$-test. (F) Transplantation model with BALB/C $(\mathrm{H}-2 \mathrm{Kd})$ as donor and $X b p 1^{\Delta \mathrm{EEC}}$ or $X b p 1^{1^{+/}}$mice as recipients, created with Biorender.com. (G) Survival of $X b p 1^{1 \mathrm{EC}}$ and $X b p 1^{1^{+/}}$mice after allogeneic hematopoietic cell transplantation (allo-HCT). Data were pooled from three independent experiments with $n=14$ mice/group. The $P$-value was calculated using the two-sided Mantel-Cox test. $(\mathrm{H})$ Histopathology scores of the small intestine and colon from $X b{ }^{1 \mathrm{ALC}}$ and $X b p 1^{1 /+}$ recipients on day 14 after allo-HCT. Data are pooled from $\mathrm{n}=5$ mice/group. Each dot represents a single mouse. The $P-$ values were calculated using the two-tailed unpaired Mann Whitney $\mathrm{U}$ test. 
were downregulated in $X b p 1^{\Delta \mathrm{IEC}}$ mice with GvHD when compared to littermate controls (Figure 4A). Amongst these were Defa1, Defa5, Defa20, Defa21, Defa22, Defa30, Defa34, Defa39, and Defa41. Defensins are anti-microbial peptides, secreted by Paneth cells. A decrease in defensin gene expression is in line with previously reported loss of Paneth cells in $X b p 1^{\triangle \mathrm{IEC}}$ mice. ${ }^{17}$ We next explored how the expression of anti-microbial peptides varied during GVHD development. We observed reduced mRNA expression of Defa1, Defa4, lysozyme $(L y z)$ and regenerating islet-derived protein $3 \gamma(R e g 3 g)$ in untreated $X b p 1^{\Delta \mathrm{IEC}}$ mice compared to $X b p 1^{+/+}$littermates. GvHD induction reduced initially the expression of all four genes (Figure 4B). On day 14 after allo-HCT, anti-microbial peptide expression in $\mathrm{Xbp1}^{+/+}$mice had returned to normal levels whereas it stayed significantly lower in Xbp1 ${ }^{\triangle \mathrm{EEC}}$ mice (Figure 4B).

Previous studies have suggested that the intestinal dysbiosis, which is frequently observed in GvHD, results at least in part from the reduction of Paneth cells and their products in intestinal crypts. ${ }^{22}$ We hypothesized that reduced anti-microbial peptide expression in $X b p 1^{\Delta \mathrm{IEC}}$ animals might be accompanied by changes in the microbiome. We analyzed the microbiome of $X b p 1^{\triangle I E C}$ and $X b p 1^{+/+}$control mice with or without GvHD induction. We observed changes on the phylum level in the untreated condition with a shift towards more Bacteroidetes and less Firmicutes in $X b p 1^{\Delta \mathrm{IEC}}$ mice (Figure $4 \mathrm{C}, \mathrm{D}$ ). Loss of Firmicutes, and specifically some Clostridia species has previously been linked to GvHD. ${ }^{23}$ We found that within the Firmicutes phylum, the Clostridia class was particularly reduced with decreased abundances of the families Ruminococcaceae and Lachnospiraceae in $X b p 1^{\Delta I E C}$ mice (Figure $4 \mathrm{E}$ ), the latter of which has been associated with a protective effect against lethal GvHD. ${ }^{24,25}$ In the early phase after allo-HCT, there were no differences in the microbiome composition between $X b p 1^{+/+}$and $X b p 1^{\Delta \mathrm{IEC}}$ mice (Figure 4C, D), mirroring the comparable levels of anti-microbial peptides that we observed at this time point (Figure 4B). These data indicate that ER stress signaling dysregulation caused by loss of $X b p 1$ induces a loss of anti-microbial peptides, coinciding with a shift in the microbiome composition in the steady-state that is equivalent to the microbiome disruption during lethal GvHD development.

\section{Loss of Xbp1 promotes a shift towards pro-apoptotic unfolded protein response signaling which can be reversed by a pharmacological intervention}

We further hypothesized that the observed aggravated GVHD phenotype might be caused by upregulation of proapoptotic UPR signaling in $X b p 1^{\triangle \mathrm{IEC}}$ mice. RIDD is an IRE1 $\alpha-$ dependent process that gets activated upon prolonged ER stress. IRE1 $\alpha$ RNase activity cleaves several mRNA with subsequent apoptosis induction. We observed signifi- cantly decreased levels of the RIDD targets bone marrow stromal cell antigen 2 (Bst2), lysosomal-associated membrane protein 1 (Lamp1), carboxylesterase 1F (Ces1f), solute carrier family 35, member B1 (Slc35b1), ribophorin II (Rpn2) and heparan- $\alpha$-glucosamide $\mathrm{N}$-acetyltransferase (Hgsnat) in the intestine of $X b p 1^{\Delta I E C}$ recipient mice (Figure $4 \mathrm{~A}$; Figure $5 \mathrm{~A}, \mathrm{~B}$ ). Consistent with the hypothesis that Xbp1 deletion favors other, pro-apoptotic ER stress branches, Ddit3, encoding for CHOP, was upregulated (Figure 5C). We then explored the question of whether compensatory over-activation of IRE1 $\alpha$-related RIDD mediates the aggravated GVHD phenotype in mice with intestinal $X b p 1$ deletion. We employed a small molecule inhibitor of IRE1 $\alpha, 4 \mu 8 \mathrm{c}$ (7-hydroxy-4-methyl-2-oxo-2H-1benzopyran-8-carboxaldehyde). This compound blocks the RNase activity of IRE1 $\alpha$, including RIDD, but spares its kinase activity. We observed that treatment of $X b p 1^{\Delta I E C}$ mice with $4 \mu 8 \mathrm{c}$ restored the survival upon GvHD induction to the level observed in $\mathrm{Xbp}^{+/+}$littermates (Figure 5D). Taken together, we show that selective inactivation of intestinal $X b p 1$ results in a major dysregulation of the UPR with a pro-apoptotic signature.

\section{Inhibition of IRE1 $\alpha$ improves graft-versus-host disease outcome without impairing immune reconstitution or the graft-versus-leukemia effect}

We observed that inhibition of IRE1 $\alpha$ reverses the toxicity of chronic ER stress in $X b p 1^{\triangle \mathrm{IEC}}$ mice and therefore studied the potential of the IRE1 $\alpha$ inhibitor as a therapeutic compound for general GVHD development and outcome. In vivo treatment of mice who had developed GvHD with the IRE1 $\alpha$ inhibitor $4 \mu 8 \mathrm{c}$ ameliorated GVHD severity, as observed by prolonged survival, reduced clinical GVHD signs and decreased histopathology score (Figure 6A, D). A beneficial effect, albeit not as strong as with $4 \mu 8 \mathrm{c}$, was observed with a second IRE1 $\alpha$ inhibitor, B-I09 (Online Supplementary Figure S4A). On the contrary, inhibition of PERK signaling did not improve the GvHD outcome (Online Supplementary Figure $S 4 B$ ). Given the beneficial effect of IRE1 $\alpha$ inhibition over PERK inhibition, we focused on the effects of the former pathway in our further analysis. In line with the hypothesis that inhibition of IRE $1 \alpha$ reduces GVHD-related intestinal apoptosis, $4 \mu 8 \mathrm{c}$ decreased intestinal cell death in response to treatment with the pro-inflammatory cytokine TNF or the ER stress inducer tunicamycin in vitro in the small intestinal cell line MODE$\mathrm{K}$ (Figure 6E, G).

Long-term success of allo-HCT strongly depends on an intact immune reconstitution that guarantees protection against pathogens and malignancy relapse. We therefore next analyzed whether IRE1 $\alpha$ inhibition had an impact on peripheral blood leukocyte and thymus regeneration (Figure 7A). We found that, as expected, hemoglobin, platelets and white blood cells were significantly decreased in the 

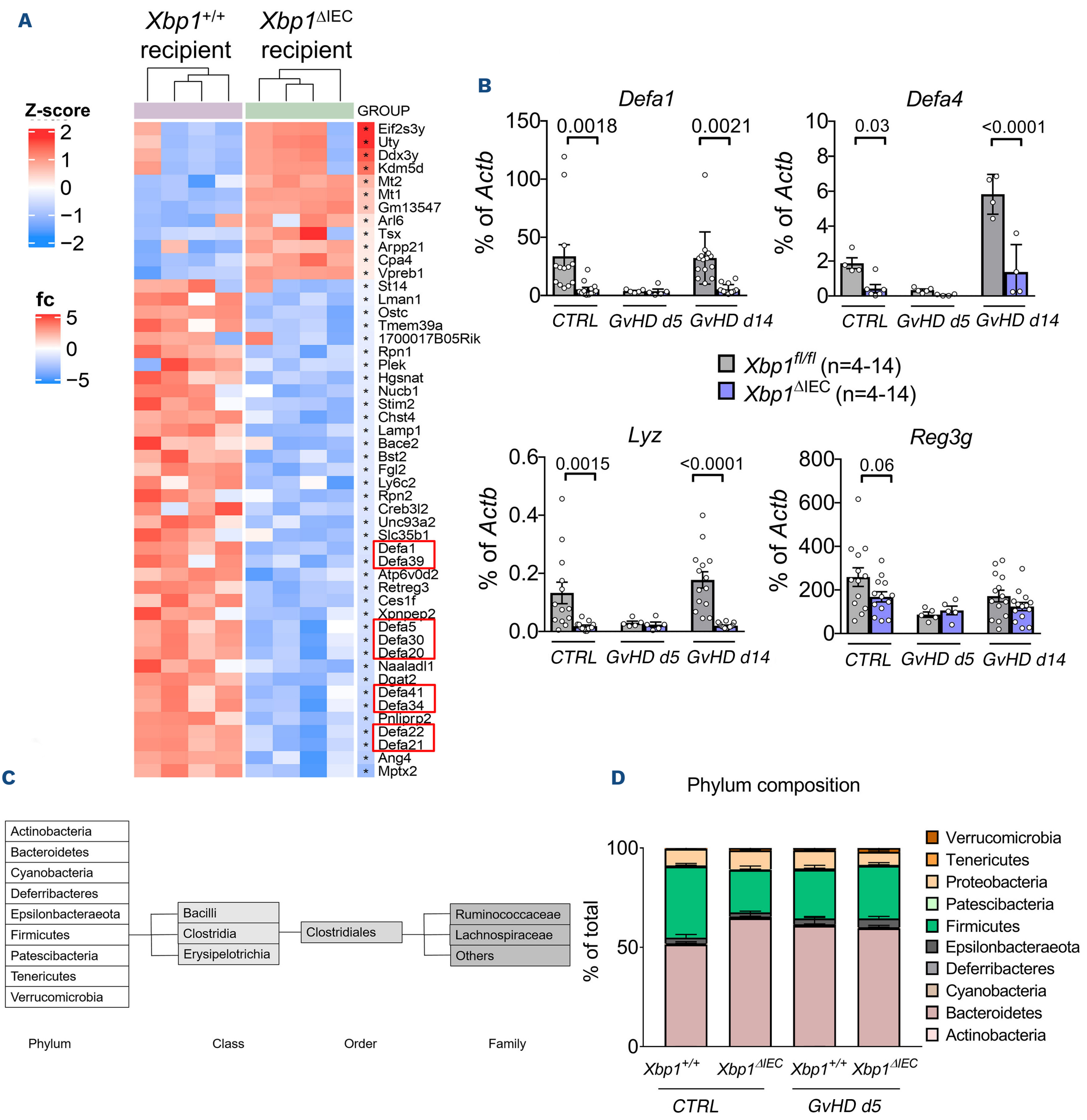

E Bacteroidetes

Firmicutes

Clostridia

Ruminococcaceae

Lachnospiraceae
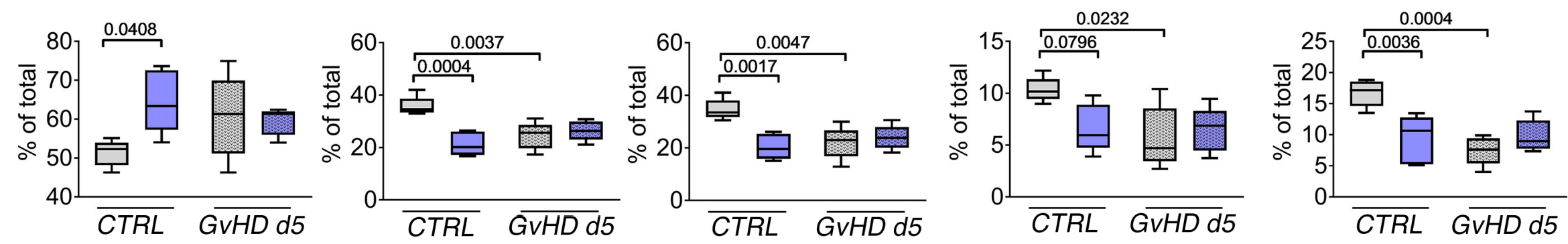

$$
\begin{aligned}
& \square \times b p 1^{f l / f l}(\mathrm{n}=5) \\
& \square \times b p 1^{\Delta \mathrm{EEC}}(\mathrm{n}=5)
\end{aligned}
$$


Figure 4. The deletion of intestinal $X b p 1$ disrupts antimicrobial peptide production and induces changes in the microbiome. (D) Heatmap showing the top 50 differentially expressed genes in the small intestine of $X b p 1^{\Delta \mathrm{EC}}$ and $X b p 1^{+/+}$recipient mice on day 14 after allogeneic hematopoietic cell transplantation (allo-HCT) performed as shown in Figure 3F. Color legend "Z-score" indicates the row-wise scaling of the normalized intensity, whereas " $\mathrm{fc}$ " indicates the $\log _{2}$ fold change between $X b p 1^{+/+}$and $X b P 1^{\triangle I E C}$ recipient mice. (B) Quantitative real-time polymerase chain reaction (PCR) analysis of the mRNA expression of the antimicrobial peptides Defa1, Defa4, Lyz and Reg $3 g$ in the indicated groups with Actb as a reference gene. Data were pooled from $\mathrm{n}=5-14$ mice/group. Each dot represents a single mouse. The $P$-values were calculated using the ordinary one-way ANOVA with correction for multiple comparisons. (C) Simplified overview of bacteria taxonomy with a focus on the phylum Firmicutes. (D, E) Microbiome analysis of untreated (CTRL) and graft-versus-host disease (GVHD)-developing $X b p 1^{1 \mathrm{LEC}}$ and $X b p 1^{1^{+/}}$mice on day 5 after allo-HCT. Data were pooled from $n=5$ independent biological replicates. The $P$-values were calculated using an ordinary one-way ANOVA with correction for multiple comparisons. (D) Phylum composition. (E) Relative abundance of the phyla Bacteroidetes and Firmicutes, the class Clostridia and the families Ruminococcaceae and Lachnospiraceae.

A

\section{$X b p 1^{\triangle \mathrm{EEC}}$ vs . Xbp $1^{+/+}$recipient}

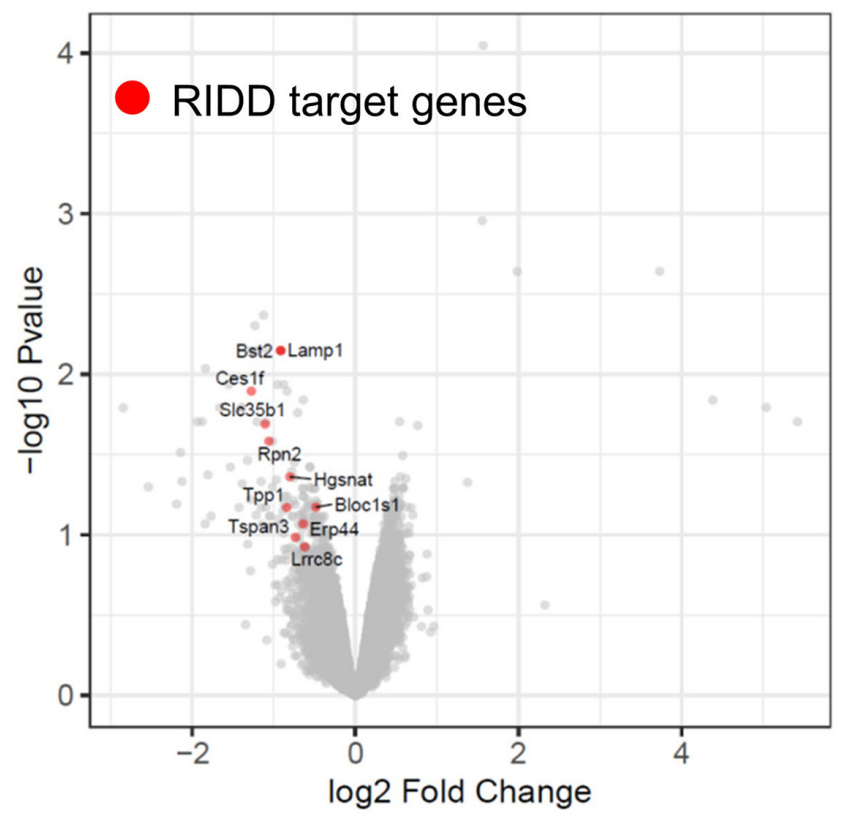

B
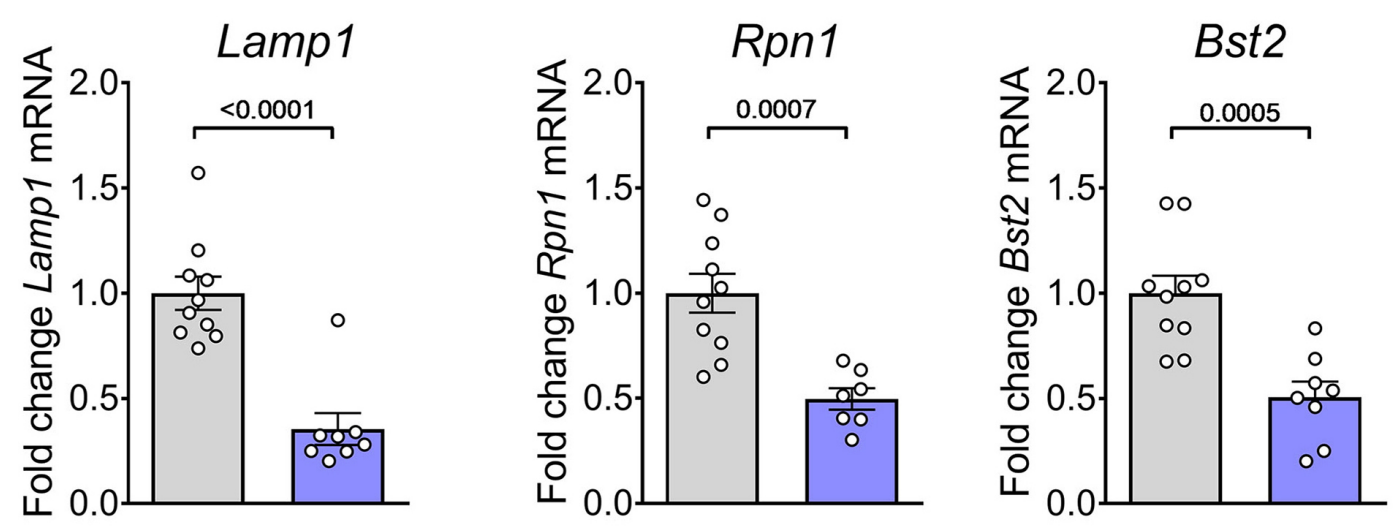

$X b p 1^{+/+}$recipient $(\mathrm{n}=10)$

$X b p 1^{\Delta \mathrm{IEC}}$ recipient $(\mathrm{n}=7)$
C

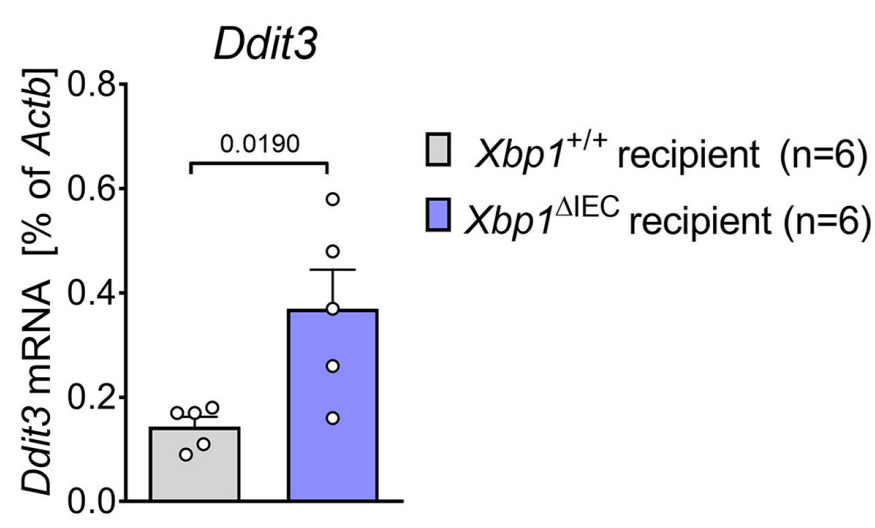

D

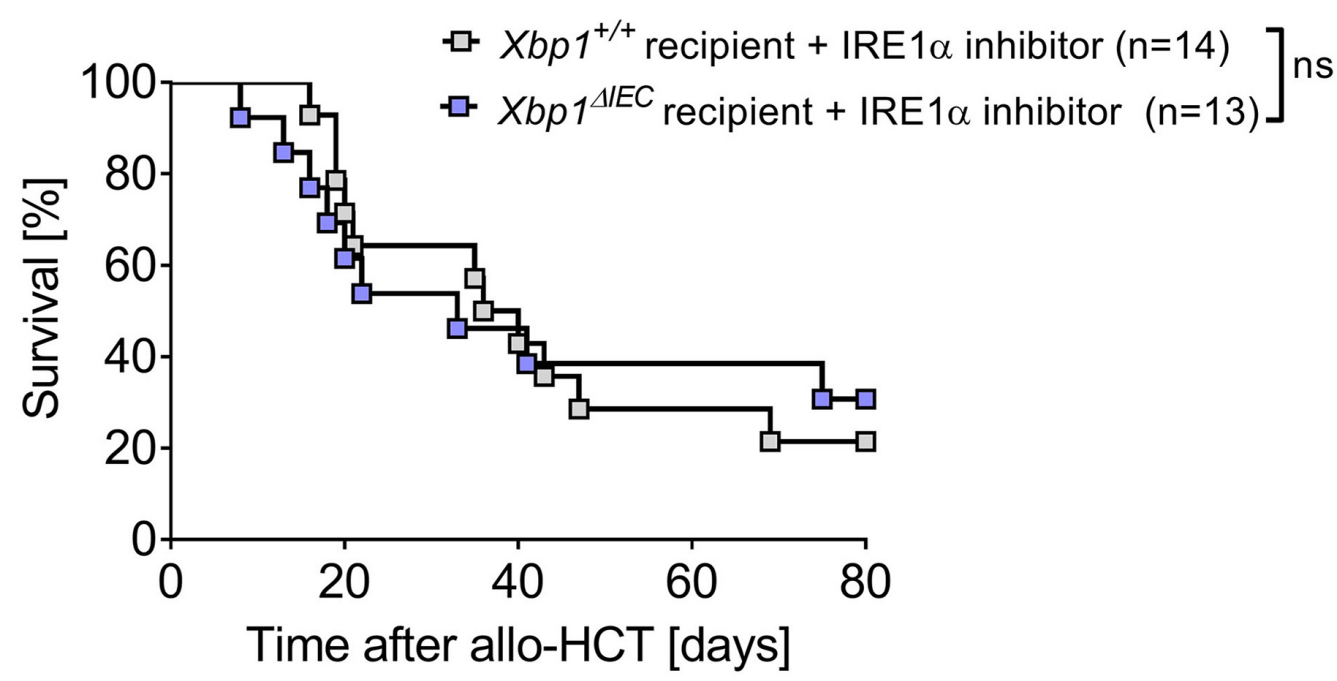

Figure 5. Loss of Xbp1 favors pro-apoptotic signaling. (A-D) $X b p 1^{\Delta \mathrm{IEC}}$ and $X b p 1^{+/+}$mice underwent bone marrow transplantation $(B M T)$ as in Figure 3F. (A) Volcano plot showing gene expression in $X b p 1^{\triangle I E C} v s . X b p 1^{+/+}$recipient mice on day 14 after allogeneic hematopoietic cell transplantation (allo-HCT). Red circles highlight mRNA that are targets of RIDD. (B) Quantitative real-time polymerase chain reaction (PCR) analysis of the mRNA expression of the RIDD target genes Lamp1, Rpn1 and Bst2 in the indicated groups with $A c t b$ as a reference gene. Data were pooled from $n=10$ mice in the $X b p 1^{+++}$group and $n=7$ mice in the $X b p 1^{\Delta I E C}$ group. Each dot represents a single mouse. $P$-values were calculated using the two-tailed unpaired Student's $t$-test. (C) Quantitative real-time PCR analysis of the mRNA expression of the pro-apoptotic endoplasmic reticulum (ER) stress gene Ddit3 (encodes for $\mathrm{CHOP}$ ) with $A c t b$ as a reference gene. Data were pooled from $\mathrm{n}=6$ mice/group. Each dot represents a single mouse. $P$-values were calculated using the two-tailed unpaired Student's $t$-test. (D) Survival of $X b p 1^{\Delta \mathrm{EC}}$ and $X b p 1^{+/+}$mice after allo-HCT and treatment with the IRE1 $\alpha$ inhibitor $4 \mu 8 \mathrm{c}$. Data were pooled from three independent experiments with $\mathrm{n}=13-14 \mathrm{mice} / \mathrm{group}$. Each dot represents a single mouse. $P$-values were calculated using the two-sided Mantel-Cox test. 
A

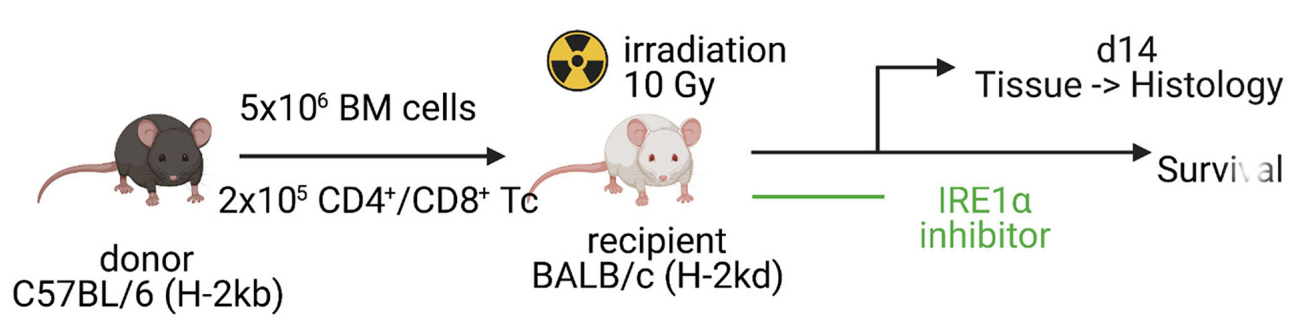

C

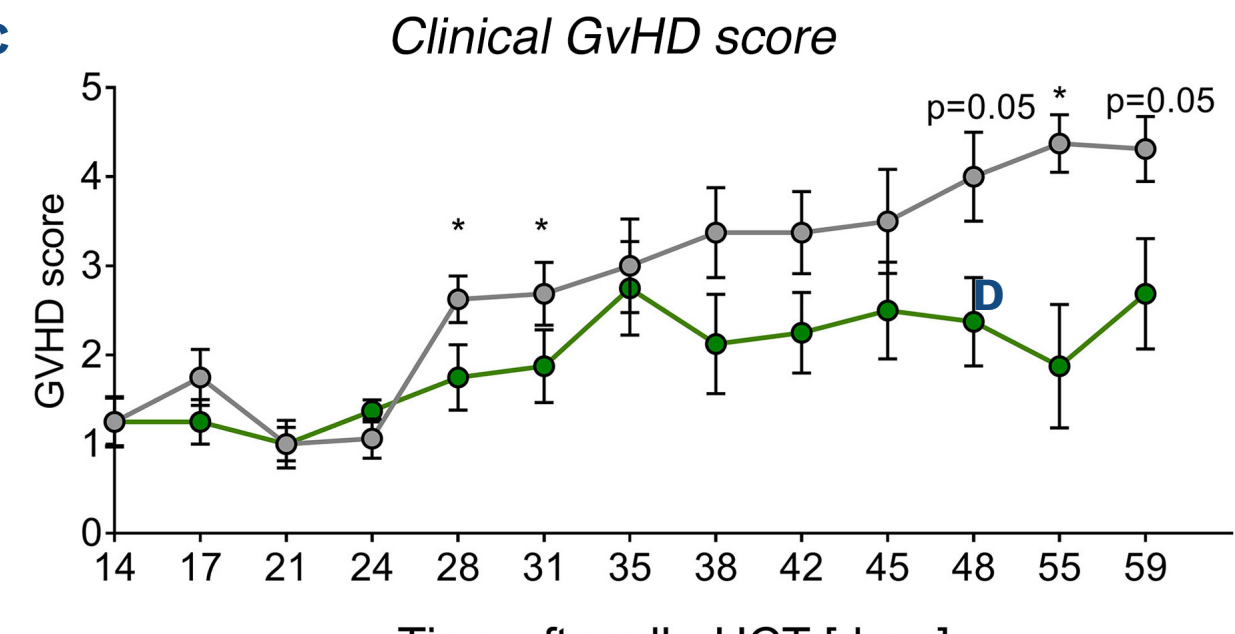

Time after allo-HCT [days]

o. $B M+T c+$ vehicle $(n=8)$

- $B M+T c+4 \mu 8 c(n=8)$
B

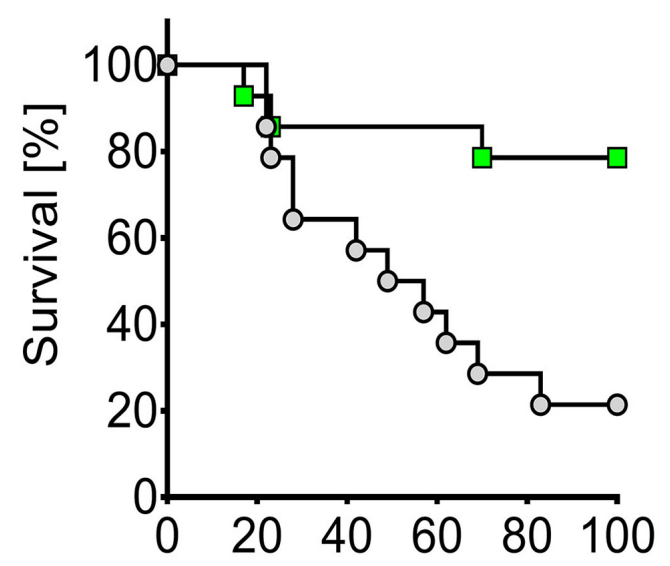

Time after allo-HCT [days]

O BM+Tc+vehicle $(n=14)$

$\square B M+T c+4 \mu 8 c(n=14)$

0.0037

D

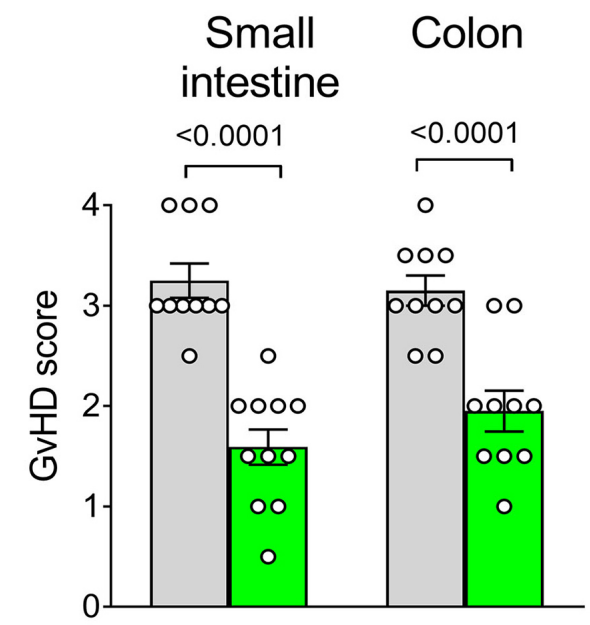

$\square$ BM+Tcells+vehicle ( $n=10)$

$\square$ BM+Tcells $+4 \mu 8 c(n=10)$

\section{E}
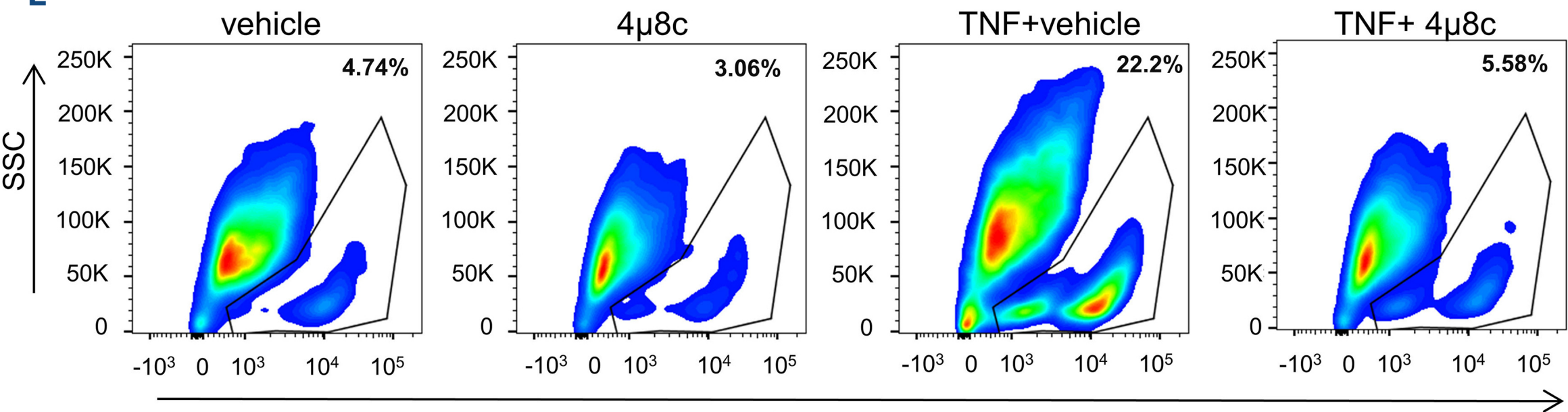

Live/Dead

F

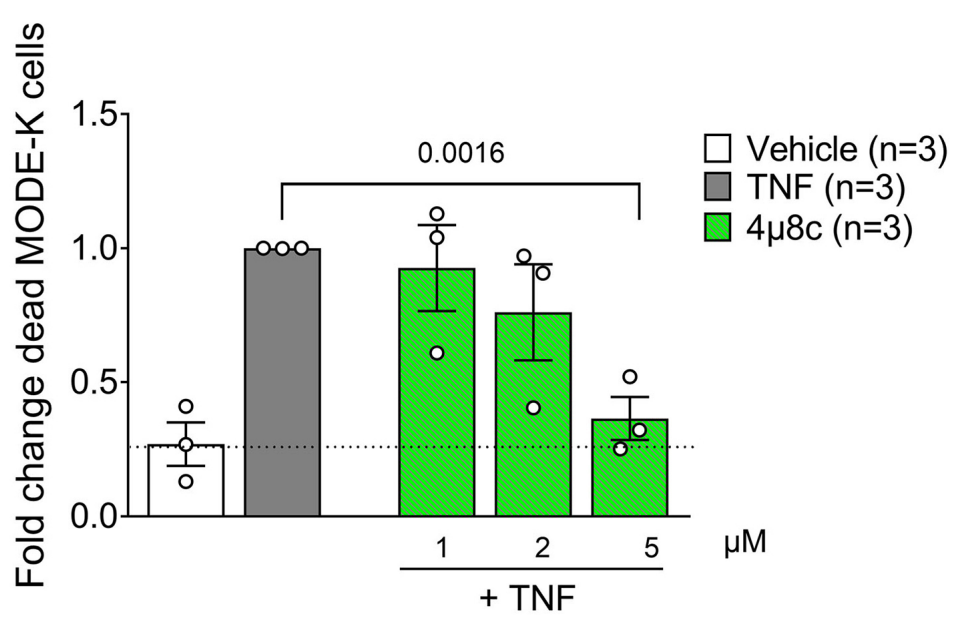

G

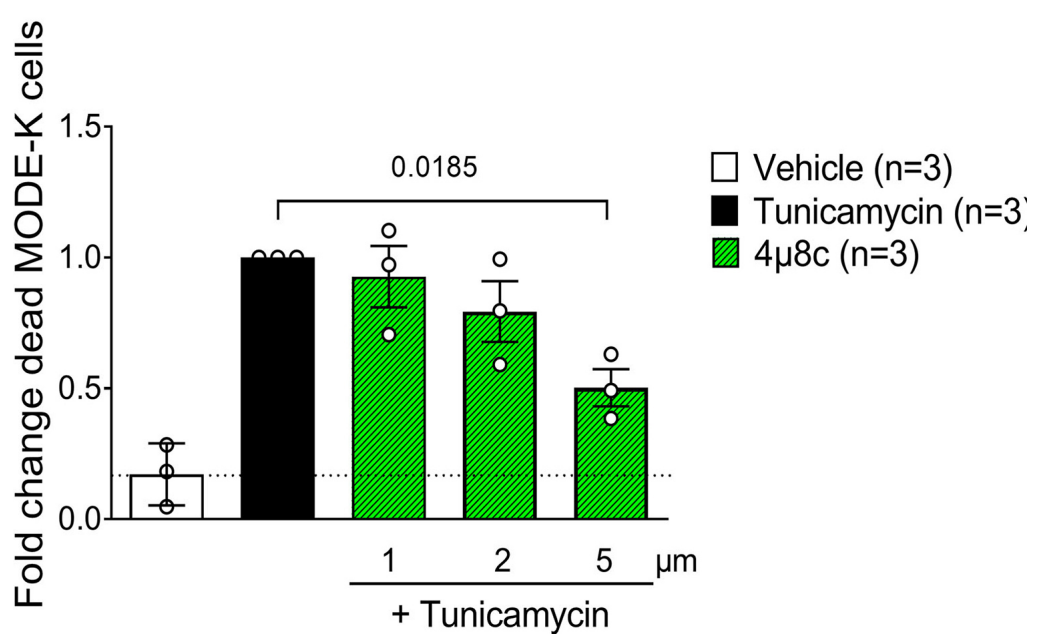

Continued on following page. 
Figure 6. Pharmacological IRE1 $\alpha$ inhibition improves graft-versus-host disease outcome in mice. (A) Transplantation model with C57BL/6 $\left(\mathrm{H}-2 \mathrm{~K}^{\mathrm{b}}\right)$ as donor and BALB/C $\left(\mathrm{H}-2 \mathrm{~K}^{\mathrm{d}}\right)$ as recipient. Recipient animals were treated with $10 \mathrm{mg} / \mathrm{kg}$ body weight IRE1 $\alpha$ inhibitor $4 \mu 8 \mathrm{c}$ (from day 0 until day 10 after bone marrow transplantation [BMT]) or an equal volume of vehicle by a daily intraperitoneal (i.p.) injection. Schematic overview created with BioRender.com. (B) Survival of BALB/c mice transplanted and treated with $4 \mu 8 \mathrm{c}$ as shown in (A). Data were pooled from two independent experiments, $\mathrm{n}=14 \mathrm{mice} / \mathrm{group}$. The $P$-value was calculated using the two-sided Mantel-Cox test. (C) Clinical graft-versus-host disease (GvHD) scores combined from weight loss, skin lesions, hunching posture, dull fur and diarrhea in BALB/c mice transplanted as shown in (A). Data were pooled from $n=8$ mice/group. The $P$-values were calculated using the two-tailed unpaired Mann-Whitney $U$ test. (D) Histopathology scores of the small intestine and colon from BALB/C mice transplanted and treated as in (A) on day 14 after allogeneic hematopoietic cell transplantation (allo-HCT). Data are pooled from $n=7(B M+v e h i c l e ~ g r o u p)$ and $n=10$ mice $(B M+T c e l l+v e h i c l e ~ a n d ~ B M+T c e l l+4 \mu 8 c$ groups). $P$-values were calculated using the two-tailed unpaired Mann-Whitney $U$ test. (E, F) Analysis of MODE-K cell viability after treatment with TNF $(20 \mathrm{ng} / \mathrm{mL}) \pm 4 \mu 8 \mathrm{c}$ (concentrations as indicated) for 48 hours (h) performed by flow cytometry. (E) Representative flow cytometry dot plots. (F) Quantification of the percentages of dead cells. Data were normalized to the TNFtreated group. Statistical analysis of $n=3$ biologically independent experiments performed in technical duplicates or triplicates. The $P$-values were calculated using the ordinary one-way ANOVA test. (G) Analysis of MODE-K cell viability after treatment with $0.15 \mu \mathrm{g} / \mathrm{mL}$ tunicamycin $\pm 4 \mu 8 \mathrm{c}$ (concentrations as indicated) for 48 hours. Quantification of the percentages of dead cells. Statistical analysis of $n=3$ independent experiments each performed in technical triplicates. Data were normalized to the tunicamycin treatment group. The $P$-values were calculated using the ordinary one-way ANOVA test.

blood of mice on day 14 after allo-HCT when compared to untreated mice. By day 29, hemoglobin and platelets had recovered to normal levels, whereas the white blood cells, and specifically $B$ and $T$ lymphocytes remained reduced by about $80 \%$ (Figure $7 \mathrm{~B}, \mathrm{C}$ ). Importantly, there was no difference between vehicle- and $4 \mu 8 \mathrm{c}$-treated animals (Figure 7B, C). Peripheral blood T-cell differentiation into naïve, effector and central memory $T$ cells was also not affected by IRE1 $\alpha$ inhibition (Online Supplementary Figure S5A). Thymus pathology following TBI and GvHD development can impair the T-cell selection process leading to the emergence of an autoreactive population and a failure to generate functional $T$ cells that fight cancer cells and pathogens. Analysis of the thymic T cell populations showed that, independently of $4 \mu 8 \mathrm{c}$ treatment, $\mathrm{CD} 4^{+} \mathrm{CD} 8^{+}$double positive (DP), that are the most frequent population in the healthy thymus, represent only $8 \%$ of the cells in allo-HCT recipients. In the same time, the single positive $\mathrm{CD}^{+}$ population had expanded to $72 \%$ (Figure 7D) after alloHCT. In the non-hematopoietic compartment, we observed no changes in the percentages of thymus epithelial cells or fibroblasts in $4 \mu 8 \mathrm{c}$ - compared to vehicle-treated animals (Figure $7 \mathrm{E}$ ). Overall, these data provide evidence that hematopoietic and thymus regeneration is not affected by administration of $4 \mu 8 \mathrm{c}$ to $\mathrm{d} 29$, although longterm studies of lymphocyte regeneration and thymic populations would be required to provide definitive confirmation.

Long-term malignancy control by allo-reactive $\mathrm{T}$ cells is essential for the success of allo-HCT. We assessed the expansion of allo-reactive $T$ cells by bioluminescence imaging and flow cytometry and found that $4 \alpha 8 \mathrm{c}$ did not impact T-cell expansion and differentiation in vivo (Figure 8A-D). We next tested whether IRE1 $\alpha$ inhibition interfered with the graft-versus-tumor effect. We activated $T$ cells via co-culture with allogeneic bone marrow-derived dendritic cells in the presence or absence of $4 \mu 8 \mathrm{c}$. After 72 hours of activation, the T cells were incubated with A20 lymphoma cells and the killing efficacy was evaluated by flow cytometry (Figure $8 \mathrm{E}$ ). We observed a similar capacity of vehicle- and $4 \mu 8 \mathrm{c}$-pretreated T cells to eliminate A20 cells (Figure 8F). Collectively, these data show that pharmacological inhibition of IRE1 $\alpha$ does not impair the killing capacity of allo-reactive T cells.

\section{Discussion}

Acute GVHD affects about $50 \%$ of allo-HCT recipients with more than $10 \%$ of all patients suffering from a severe course of the disease." Recent efforts to improve the efficacy of treatment and prophylaxis range from blockade of T-cell activation,,$^{26,27}$ to inhibition of cytokine signaling ${ }^{28,29}$ and epigenetic therapies. ${ }^{30}$

In this study, we hypothesized that intestinal ER stress could be a target for novel GvHD therapies. We observed an upregulation of multiple ER stress markers following irradiation and GVHD induction. This is in line with a previous report showing upregulation of chaperones after allogeneic transplantation. ${ }^{31}$ Upregulation of ER stress markers has also been observed in the lacrimal gland, small intestine, skin and liver of mice developing chronic GvHD. ${ }^{32}$ In an extended analysis of a publicly available RNA sequencing data set (GSE134662), ${ }^{18}$ we found increased expression of several UPR genes in human GVHD samples compared to healthy tissue. Furthermore, we found that the expression of two ER stress markers, GRP78 and CHOP, directly correlated with GVHD severity in a cohort of patients at our center. The association of ER stress marker expression with higher-grade GVHD in patients strengthens the implication that modulation of intestinal UPR might be a successful treatment approach.

We next hypothesized that chronic unresolved ER stress might aggravate GVHD. We performed experiments using murine intestinal organoids as an in vitro model, and with a conditional knockout model of the transcription factor Xbp1 in vivo. Induction of ER stress by a chemical compound in- 
A

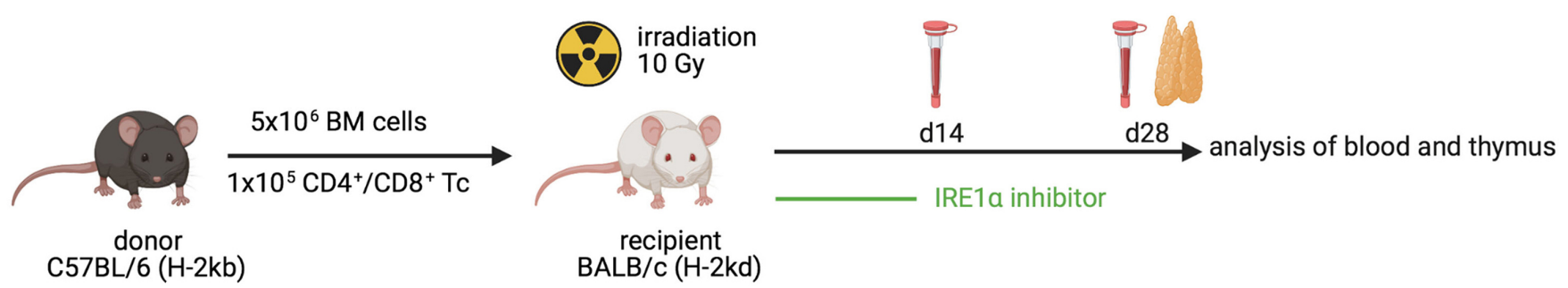

B

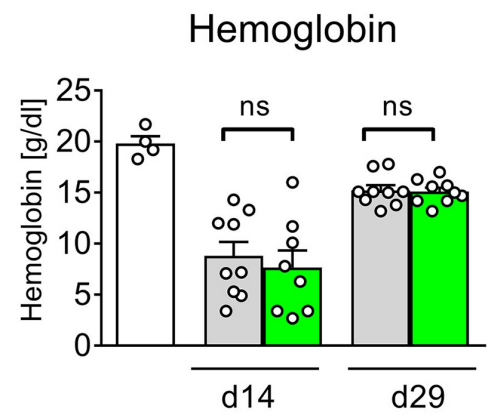

Granulocytes

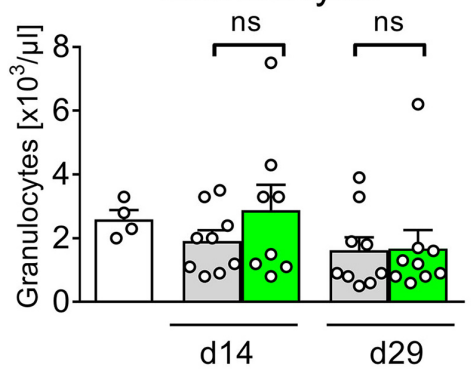

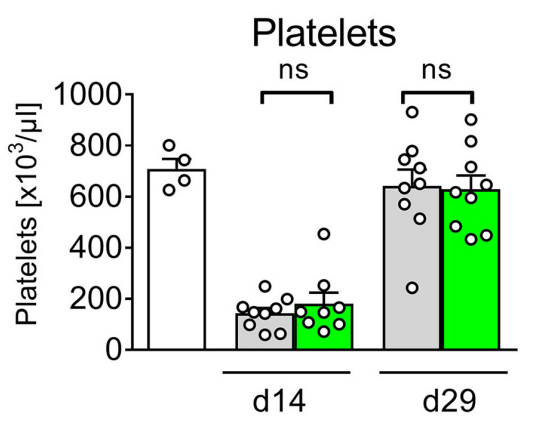

Monocytes

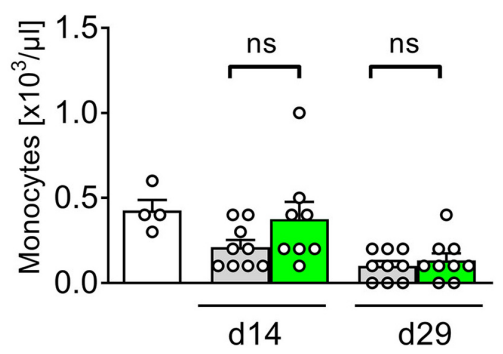

White blood cells

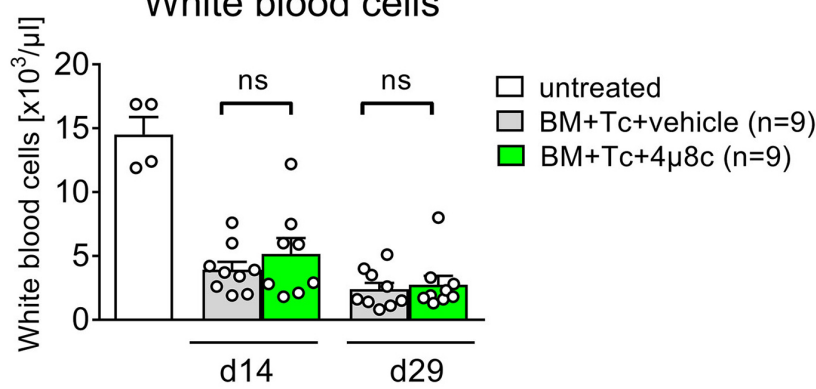

Lymphocytes

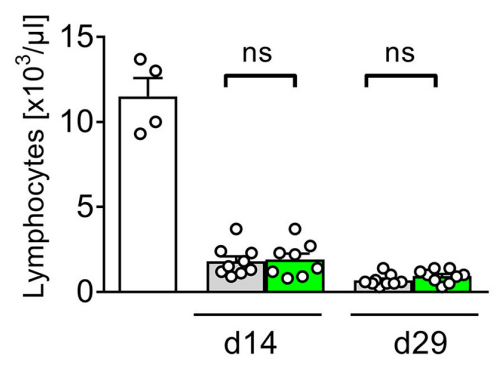

C
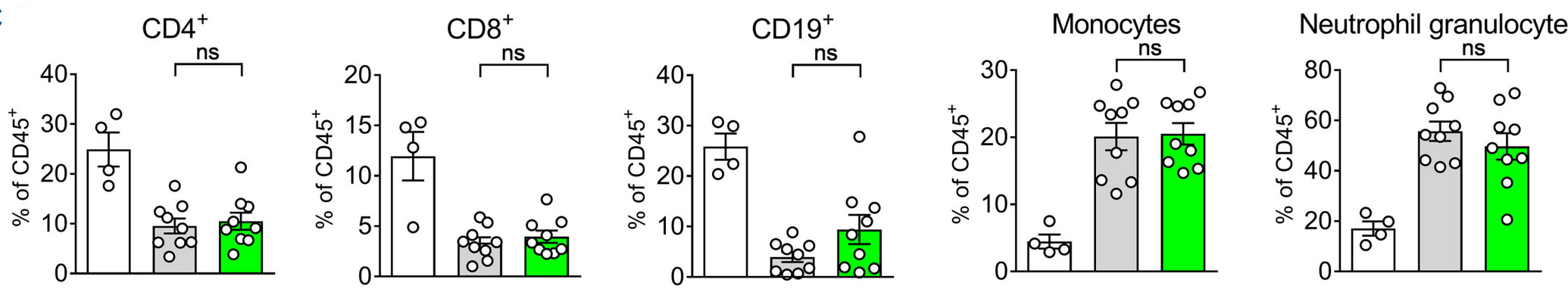

D

Thymic T cell populations
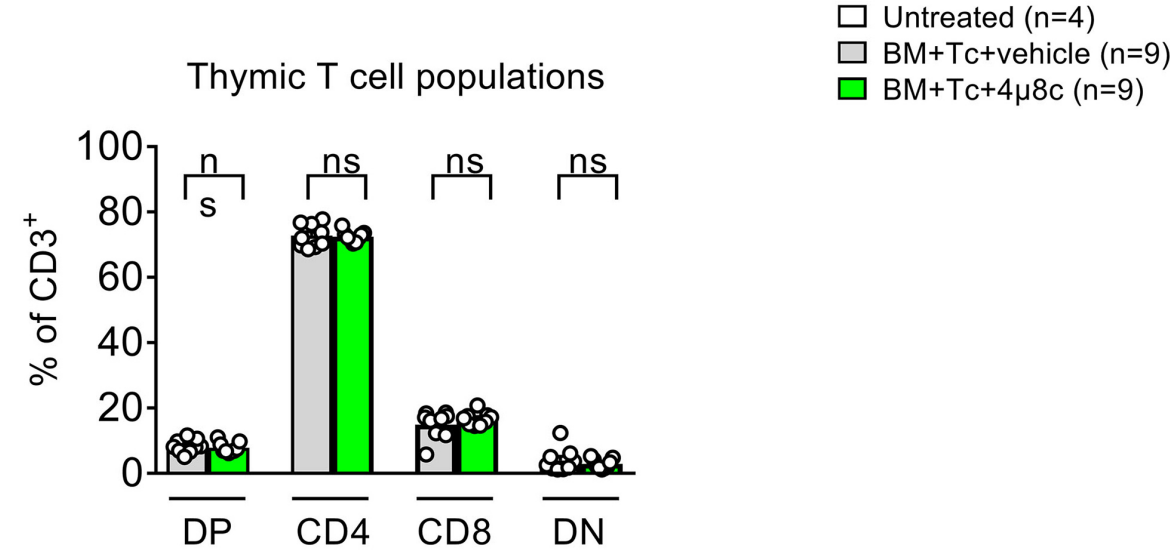

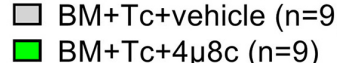

E

E Non-hematopoietic compartment

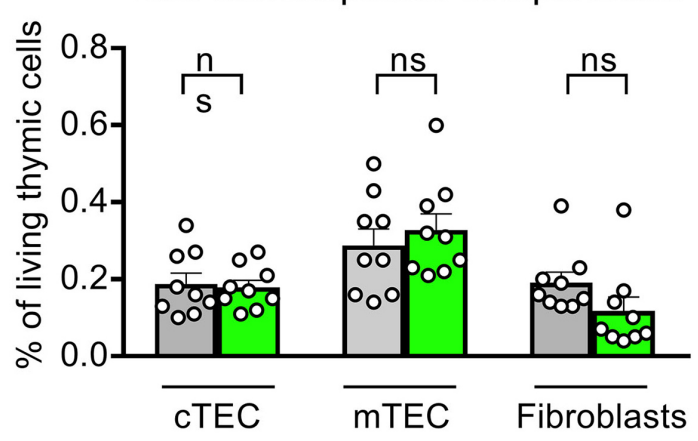

BM+Tc+vehicle $(n=9)$

$B M+T c+4 \mu 8 c(n=9)$

Figure 7. IRE1 $\alpha$ inhibition does not impair thymic and peripheral blood regeneration. (A-E) BALB/C mice underwent transplantation and were treated with $4 \mu 8 \mathrm{c}$ as described in Figure $6 \mathrm{~A}$. On day 14 and day 29 after allogeneic hematopoietic cell transplantation (allo-HCT), leukocyte subpopulations and phenotype in the peripheral blood was analyzed. In addition, thymus reconstitution was assessed on day 29. Data were pooled from $n=4$ mice in the untreated group and $n=9$ mice each for the other groups. Each dot represents a single mouse. P-values were calculated using the two-tailed unpaired Student's $t$-test. ns: not significant. (A) Schematic overview of the experiment, created with BioRender.com. (B) Hemoglobin, platelet and white blood cell count including differentiation into granulocytes, monocytes and lymphocytes from mice treated as shown in (A). (C) Percentages of leukocyte subpopulations assessed by flow cytometry. Pre-gating on single cells - living cells - CD45 ${ }^{+}$was performed. Neutrophils were defined as CD11 $\mathrm{b}^{+}{\text {Ly } 6 G^{+}}^{\text {. Monocytes were defined as CD11 }{ }^{+} \text {Ly6G }}{ }^{-}$. (D) Percentages of double positive (DP, $C D 4^{+} C D 8^{+}$), single $C D 4^{+}$, single $C D 8^{+}$and double negative (DN, CD4-CD8-) $T$ cells in the thymus of recipient mice. Pre-gating on single cells- living cells - CD45 $5^{+}-\mathrm{CD}^{+}$was performed. (E) Percentages of medullary thymic epithelial cells (mTEC, CD45- EpCAM $^{+}$Ly51 $^{+}$), cortical thymic epithelial cells (cTEC, CD45- EpCAM ${ }^{+}$Ly51-) and fibroblasts (CD45-CD140 $\left.{ }^{+}\right)$in the thymus of recipient cells. Pre-gating on single cells and living cells was performed. 
A

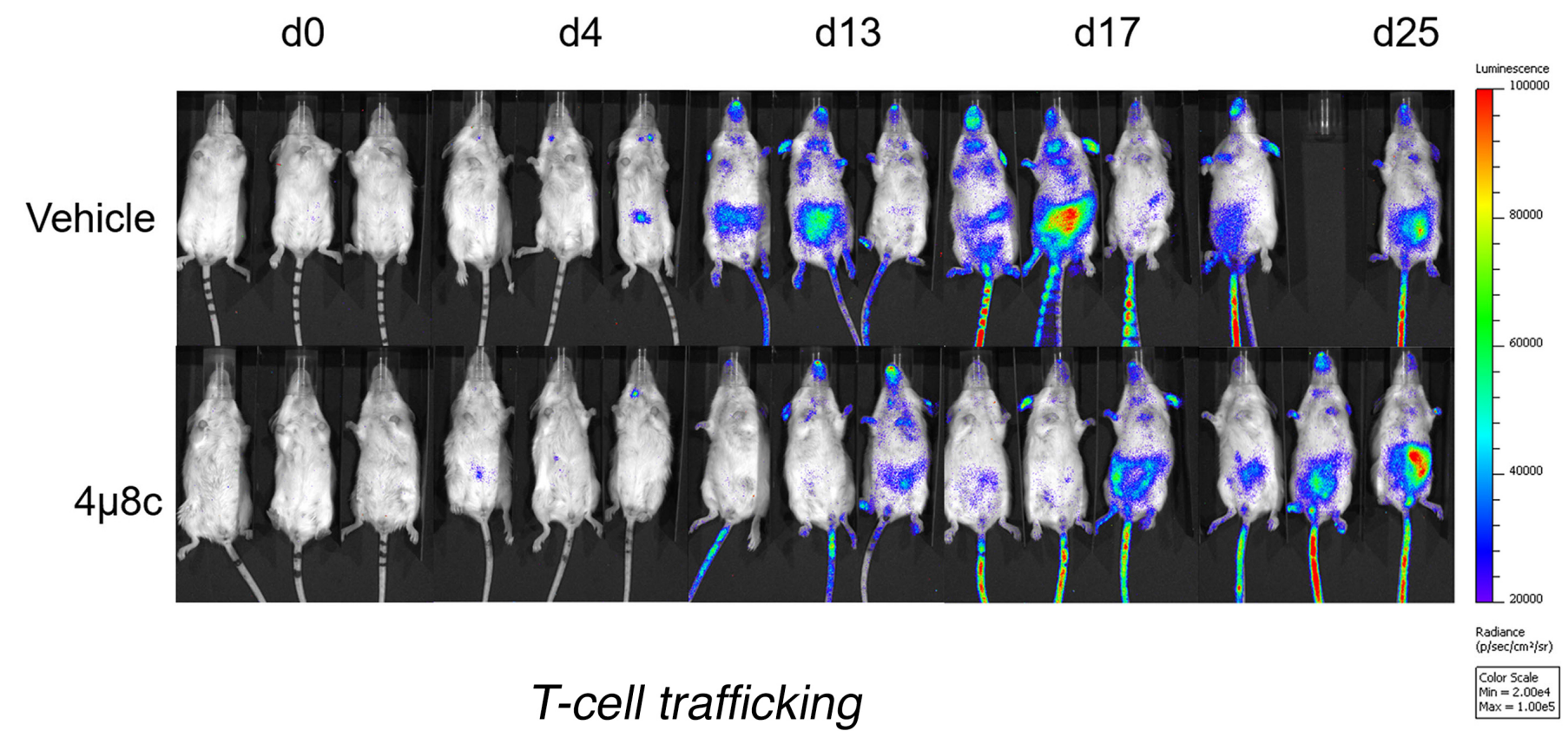

B

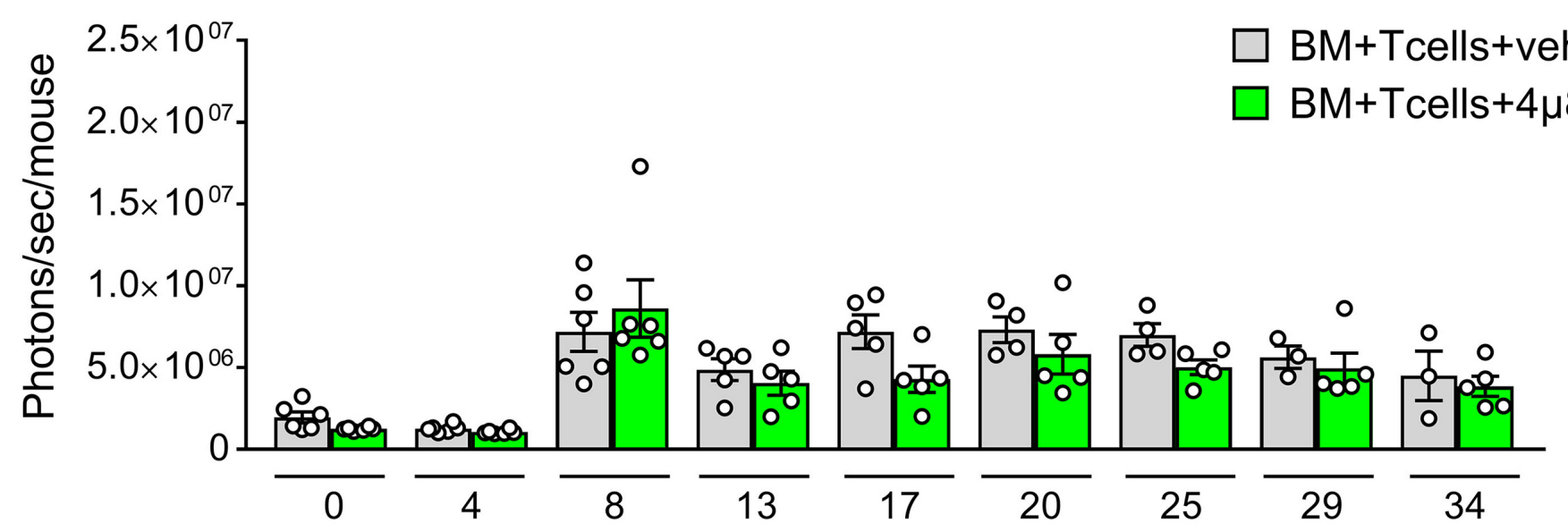

Time after allo-HCT [days]

$$
\text { C }
$$

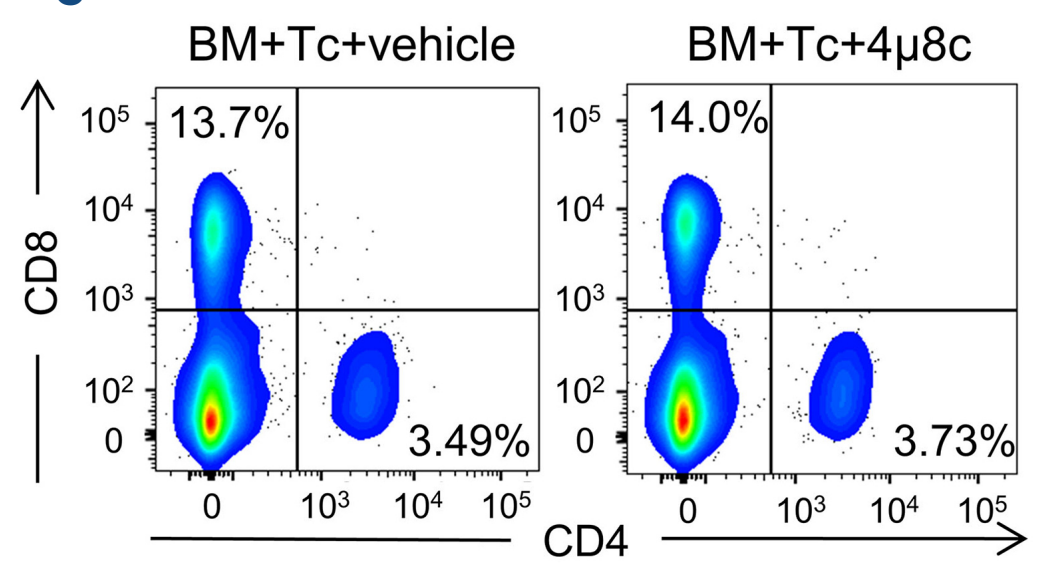

E
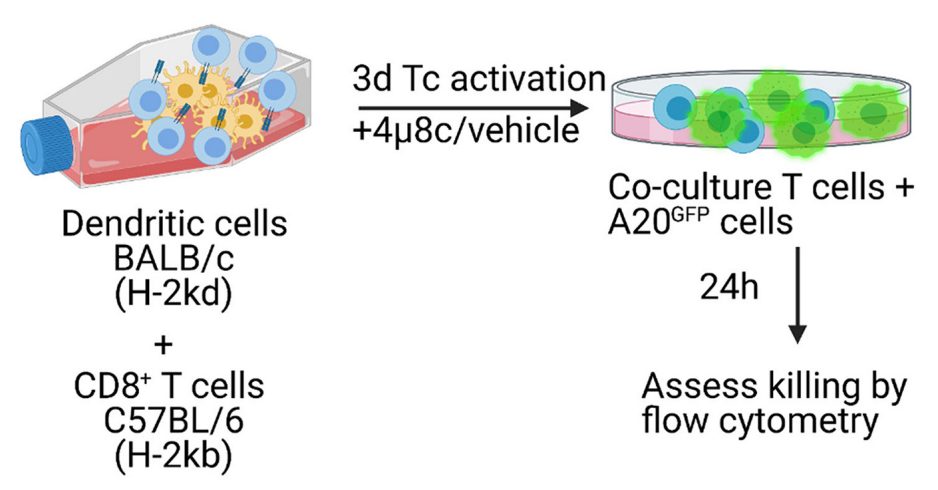

D
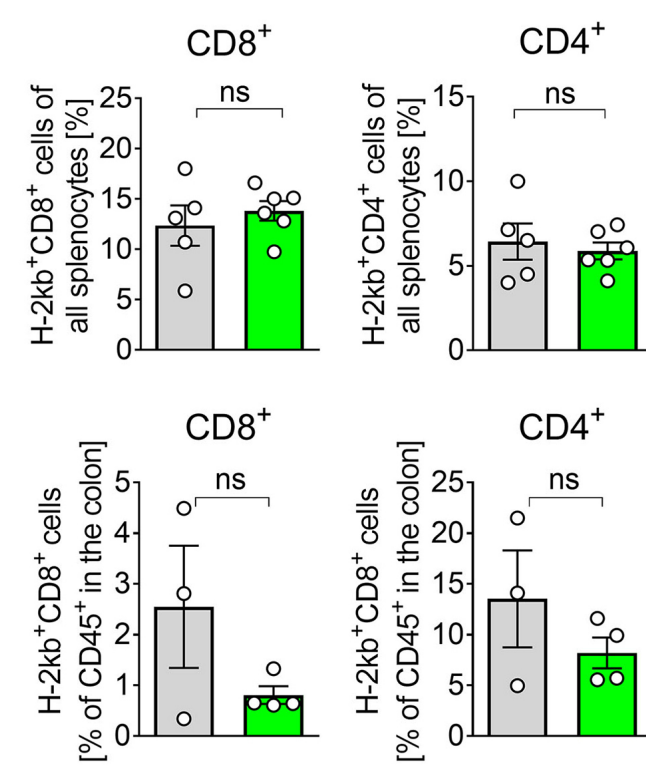

C BM+Tc+vehicle $(\mathrm{n}=3-5)$ $\square \mathrm{BM}+\mathrm{TC}+4 \mu 8 \mathrm{C}(\mathrm{n}=3-6)$

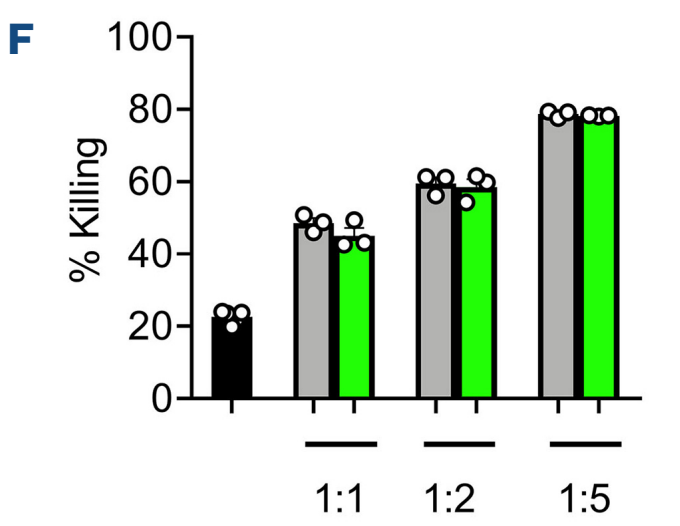

A20 only $\square$ A20+Tc (vehicle) $\square$ A20+Tc $(4 \mu 8 \mathrm{c})$

A20:T cell ratio 
Figure 8. IRE1 $\alpha$ inhibitor treatment does not affect alloreactive T-cell expansion. (A, B) BALB/c mice underwent transplantation as described in Figure 6A using luciferase-transgenic T cells. (A) Representative bioluminescence (BLI) images from several time points. (B) Quantification of the BLI measurement is shown. Signal was quantified from the whole body in photons/sec/mouse. Data were pooled from $n=6$ mice/group. Each dot represents a single mouse. One representative result from three independent experiments is shown. (C, D) Flow cytometry analysis of T cells isolated from recipient spleens and colons on day 14 after allogeneic hematopoietic cell transplantation (allo-HCT) (C57BL/6 in BALB/c model). (C) Representative flow cytometry dot plots from the spleen are shown. (D) Combined data pooled from $n=5-6$ mice/group for the percentage of donor $C D 4^{+}$and $C D 8^{+} T$ cells in the spleen (upper panel) and colon lamina propria (lower panel). Each dot represents a single mouse. One representative result from two independent experiments is shown. $P$-values were calculated using the two-tailed unpaired Student's $t$-test. ns: not significant. (E) C57BL/6 CD8 ${ }^{+} \mathrm{T}$ cells were activated in a co-culture with allogeneic BALB/C bone marrow-derived dendritic

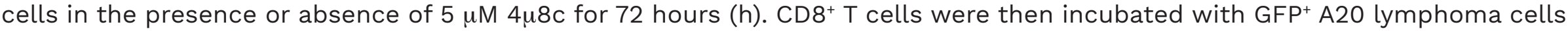
and the percentage of dead cells after $24 \mathrm{~h}$ of culture was assessed by flow cytometry. Schematic overview created with BioRender.com. (F) Percentage of dead A20 cells alone or at three different co-culture ratios with CD8 ${ }^{+} \mathrm{T}$ cells activated as described in (E). The experiment was repeated three times with four or five replicates each. One representative result is shown.

duced cell death in intestinal organoids with an increased release of the DAMP uric acid. Production of uric acid as a consequence of tissue damage during the conditioning treatment has been shown to promote GvHD by activating antigen-presenting cells. ${ }^{20,21}$ Short-term ER stress induces a stress-associated transcriptomic reprogramming with genes related to cellular signaling, expression regulation, metabolism, the cytoskeleton and others being upregulated. ${ }^{33}$ Among these are multiple inflammation-associated genes, in particular chemokines, cytokines and acute phase proteins. ${ }^{33}$ Higher expression of these mediators due to chronic activation of the UPR might perpetuate the tissue damage during GvHD by supporting the characteristic inflammatory microenvironment of the disease.

Deletion of Xbp1 led to a global dysregulation of the UPR in mice developing GVHD and was associated with a significantly decreased survival rate and increased GVHD histopathology. In a preclinical model of liver injury, mice with liver-specific deletion of Xbp1 showed initially a similar UPR activation after chemical ER stress induction with tunicamycin when compared to wild-type animals. However, this activation was significantly prolonged indicating the development of chronic ER stress in these animals. ${ }^{34}$ UPR dysregulation by Xbp1 deletion changes the composition of the intestinal epithelium. Previous studies by Kaser et al. show that mice with a Xbp1 deletion in the intestinal epithelium had a loss of Paneth and goblet cells with rising age while at the same time intestinal stem cells and transitamplifying cells appeared increased. ${ }^{17,35}$ Upon GvHD induction, a loss of Paneth cell occurs and its severity is a predictive marker of GVHD outcome. ${ }^{22,36,37}$ In our study, this loss was more pronounced in animals with a selective deletion of Xbp1 with multiple defensin subtypes, which are produced by Paneth cells, being amongst the most differentially regulated genes in $X b p 1^{1 \mathrm{EC}}$ mice. One additional mechanism by which ER stress might modulate intestinal inflammation is by altering the microbiome. The microbiome has become increasingly important for the understanding of GVHD pathology. Several studies in mice and humans show that GvHD severity and mortality are correlated with reduced microbial diversion and a shift from protective towards det- rimental bacterial species. ${ }^{38,39}$ A protective role has been shown for the phylum Firmicutes and specifically for members of the Clostridia class. ${ }^{24,25}$ The beneficial effects of these bacteria could be attributed to the generation of antiinflammatory metabolites, such as short-chain fatty acids. To the best of our knowledge, this study is the first one to describe the intestinal microbiome composition in Xbp1 ${ }^{\Delta I E C}$ mice. We observed that these mice have reduced abundancy of Firmicutes compared to their wild-type littermates. In particular, the Clostridia class including the Lachnospiraceae family were decreased. These changes closely resemble the changes observed during GvHD induction. One possible explanation for this dysbiosis is, that the intestinal environment is changed due to the reduced numbers of Paneth cells, and the lower concentrations of their anti-microbial products, defensins, lysozyme and Reg3 $\gamma$. In our view, these data support the hypothesis that there are pre-existing changes in the microbiome in $X b p 1^{\Delta \mathrm{EC}}$ mice that predispose to GVHD development.

The UPR provides multiple targets for signaling modulation by inhibition of specific branches. We proposed that specific UPR signaling inhibition might aid in modulating ER stress signaling from pro-apoptotic to cell-protective pathways. Here, we used $4 \mu 8 \mathrm{C}$ and B-I09, inhibitors of IRE1 $\alpha$ RNase activity, and GSK2606414 as a PERK inhibitor. Administration of IRE1 $\alpha$ inhibitors, but not the PERK inhibitor, showed a beneficial effect in GVHD-developing mice. Mechanistically, $4 \mu 8 \mathrm{c}$ decreased TNF-associated intestinal cell death. A crosstalk between IRE1 $\alpha$ and TNF signaling has been documented previously..$^{40}$ ER stress can induce the expression of various pro-inflammatory cytokines ${ }^{41}$ Indeed, administration of $4 \mu 8 \mathrm{c}$ in a murine model of rheumatoid arthritis decreased disease severity by blocking pro-inflammatory cytokine secretion. ${ }^{42}$ At the same time, TNF is known to induce ER stress..$^{43}$ It is conceivable that pro-apoptotic UPR employs at least in part the same pathways as TNF signaling so that inhibition of IRE1 $\alpha$ can ameliorate the cytotoxic effects of both. Interestingly, $4 \mu 8 \mathrm{c}$ showed a higher protective effect in GVHD animals, compared to B-I09. These differences might be due to variations in bioavailability after intraperitoneal injection. Another potential consideration would be 
their specificity. Both compounds have been developed as potent and specific inhibitors of IRE1 $\alpha$ RNase activity, and little is known about potential off-target effects. In a previous study, $4 \mu 8 \mathrm{c}$ was reported to have anti-oxidant capacity in endothelial and pancreatic cells by inhibiting xanthine/xanthine oxidase-catalyzed superoxide production and angiotensin II-induced ROS production. ${ }^{44}$ In light of this report, it is conceivable that $4 \mu 8 \mathrm{c}$ has combined beneficial effects in GVHD due to IRE1 $\alpha$ inhibition, and an anti-oxidative effect, which makes it a particularly interesting compound for further testing.

Besides having influence on signaling in intestinal cells, systemic IRE1 $\alpha$ inhibition might potentially have effects on immune cell reconstitution and immune responses against pathogens and malignant cells. Our data show that $4 \mu 8 \mathrm{c}$ did neither cause alterations in platelet and hemoglobin recovery, nor on lymphocyte differentiation and effector memory development. Furthermore, we did not observe a change in T-cell expansion and cytokine production upon administration of $4 \mu 8 \mathrm{c}$. The capacity to kill A20 lymphoma cells was also not affected. These data are in line with previous reports, showing that dendritic cells, which were activated in the presence of the IRE1 $\alpha$ inhibitor B-109, were capable of eliciting strong anti-tumor responses in T cells. ${ }^{45}$ In addition, a direct anti-tumor effect of B-109 against chronic lymphocytic leukemia cells has been reported. ${ }^{46} \mathrm{In}$ terestingly, specific inhibition of IRE1 $\alpha$ significantly inhibited influenza A viral replication in cell lines. ${ }^{47}$ In light of these publications, our results provide encouraging evidence that $4 \mu 8 \mathrm{c}$ treatment might spare anti-viral and anti-tumor responses while diminishing GvHD severity, even though further in vivo studies are necessary to provide additional confirmation.

Contrary to acute GVHD pathogenesis, donor B cells play an essential role in the context of chronic GvHD. These cells secrete antibodies in high quantities and are therefore highly dependent on a properly functioning UPR and are sensitive to ER stress; additionally it was found that XBP1 is essential for plasma cell differentiation. ${ }^{48} \mathrm{It}$ is therefore conclusive that several attempts were made to target ER stress and the UPR in the setting of chronic GVHD. The administration of the IRE1 inhibitor B-109 was found to reduce clinical features in a cutaneous model of chronic GvHD, with infiltrations of the skin by donor $T$ cells and dendritic cells being reduced. ${ }^{49}$ In another study, the use of the chemical chaperone 4-phenylbutyric acid (4-PBA) led to the amelioration of chronic GvHD-induced fibrosis. ${ }^{32}$ Based on our data, we propose that excessive ER stress and the activation of the UPR are mechanisms which mediate tissue injury during intestinal GVHD. Our study provides the first evidence that administration of an IRE1 $\alpha$ inhibitor is a pharmacologi- cal intervention that reduces intestinal GVHD in mice and should be considered for testing in GVHD patients.

\section{Disclosures}

$R Z$ received speakers fees from Novartis, Incyte and Mallinckrodt.

\section{Contributions}

$E H, R Z$ and $P A$ developed the overall concept and designed research; $E H, F M U, B S, D P$, and $P A$ conducted the experiments and analyzed data; $G A$ and $M B$ performed bioinformatics analysis; MK, MP and BG performed and analyzed 16S rRNA sequencing; ASG and KA developed and analyzed histological stains; PE, SM, NK and JD provided reagents and/or conceptual input; $P A$ and $R Z$ provided funding and supervision; $E H$ and PA wrote the manuscript. All authors discussed the results and contributed to the final manuscript.

\section{Acknowledgments}

The authors would like to acknowledge Dr. L. Glimcher for providing the XBP1 flox/flox mice as a gift; Dr. D. Kaiserlian for providing the MODE-K cell line; Dr. H. Andrlova for providing protocols for experimental procedures; and K. Gräwe for performing immunohistochemical stainings.

\section{Funding}

PA was supported by the Else Kröner-Fresenius-Stiftung (EKFS 2015_A147 to PA), the German Cancer Consortium (DKTK, FR 01-375) and a scholarship from the Berta Ottenstein Program for Clinician Scientists, Faculty of Medicine, Medical Center University of Freiburg, Germany. $R Z$ is supported by the Deutsche Forschungsgemeinschaft (DFG): SFB1479 (Project ID: 441891347), SFB1160, TRR167 and SFB850, the INTERREG $V$ European regional development fund (European Union) program (Project 3.2 TRIDIAG), the European Union: GVHDCure Proposal $n^{\circ} 681012$ ERC consolidator grant, the Deutsche Krebshilfe (grant number 70113473), the Jose-Carreras Leukemia foundation (grant number DJCLS 01R/2019) and the Wilhelm Sander Stiftung (grant 2008.046.4). NK was supported by the German Research Foundation (DFG) under German's Excellence Strategy (CIBSS - EXC 2189 - Project ID 390939984). MB is supported by the Deutsche Forschungsgemeinschaft (DFG) - SFB 850 subprojects C9 and Z1, SFB1479 (Project ID: 441891347- S1), SFB1160 (Project Z02), SFB1453 (Project S1) and TRR167 (Project Z01), the German Federal Ministry of Education and Research by MIRACUM within the Medical Informatics Funding Scheme (FKZ 01ZZ1801B).

\section{Data-sharing statement}

Microarray data are available on GEO under accession number GSE156469 (with the token uhgraykubhmppul). 


\section{References}

1. Zeiser R, Blazar BR. Acute graft-versus-host disease - biologic process, prevention, and therapy. N Engl $J$ Med. 2017;377(22):2167-2179.

2. Zeiser R. Advances in understanding the pathogenesis of graftversus-host disease. Br J Haematol. 2019;187(5):563-572.

3. Köhler N, Zeiser R. Intestinal microbiota influence immune tolerance post allogeneic hematopoietic cell transplantation and Intestinal GVHD. Front Immunol. 2018;9:3179.

4. Malard F, Huang XJ, Sim JPY. Treatment and unmet needs in steroid-refractory acute graft-versus-host disease. Leukemia. 2020;34(5):1229-1240.

5. Lindemans CA, Calafiore M, Mertelsmann AM, et al. Interleukin22 promotes intestinal-stem-cell-mediated epithelial regeneration. Nature. 2015;528(7583):560-564.

6. Zhang $X$, Liu $S$, Wang $Y$, et al. Interleukin22 regulates the homeostasis of the intestinal epithelium during inflammation. Int J Mol Med. 2019;43(4):1657-1668.

7. Takashima S, Kadowaki M, Aoyama K, et al. The Wnt agonist Rspondin1 regulates systemic graft-versus-host disease by protecting intestinal stem cells. J Exp Med. 2011;208(2):285-294.

8. Hayase E, Hashimoto D, Nakamura K, et al. R-Spondin1 expands Paneth cells and prevents dysbiosis induced by graft-versushost disease. J Exp Med. 2017;214(12):3507-3518.

9. Norona J, Apostolova P, Schmidt D, et al. Glucagon-like peptide 2 for intestinal stem cell and Paneth cell repair during graftversus-host disease in mice and humans. Blood. 2020;136(12):1442-1455.

10. Haring E, Uhl FM, Andrieux G, et al. Bile acids regulate intestinal antigen presentation and reduce graft-versus-host disease without impairing the graft-versus-leukemia effect. Haematologica. 2021;106(8):2131-2146.

11. Hetz C, Chevet E, Harding HP. Targeting the unfolded protein response in disease. Nat Rev Drug Discov. 2013;12(9):703-719.

12. Calfon M, Zeng $H$, Urano F, et al. IRE1 couples endoplasmic reticulum load to secretory capacity by processing the XBP-1 mRNA. Nature. 2002;415(6867):92-96.

13. Yoshida H, Matsui T, Yamamoto A, Okada T, Mori K. XBP1 mRNA is induced by ATF6 and spliced by IRE1 in response to ER stress to produce a highly active transcription factor. Cell. 2001;107(7):881-891.

14. Nishitoh H, Matsuzawa A, Tobiume K, et al. ASK1 is essential for endoplasmic reticulum stress-induced neuronal cell death triggered by expanded polyglutamine repeats. Genes Dev. 2002;16(11):1345-1355.

15. Urano F, Wang $X$, Bertolotti A, et al. Coupling of stress in the ER to activation of JNK protein kinases by transmembrane protein kinase IRE1. Science. 2000;287(5453):664-666.

16. Han D, Lerner AG, Vande Walle L, et al. IRE1alpha kinase activation modes control alternate endoribonuclease outputs to determine divergent cell fates. Cell. 2009;138(3):562-575.

17. Kaser A, Lee AH, Franke A, et al. XBP1 links ER stress to intestinal inflammation and confers genetic risk for human inflammatory bowel disease. Cell. 2008;134(5):743-756.

18. Holtan SG, Shabaneh A, Betts BC, et al. Stress responses, M2 macrophages, and a distinct microbial signature in fatal intestinal acute graft-versus-host disease. JCI Insight. 2019;5(17):e129762.

19. Sato T, Clevers H. Primary mouse small intestinal epithelial cell cultures. Methods Mol Biol. 2013;945:319-328.

20. Wilhelm K, Ganesan J, Muller T, et al. Graft-versus-host disease is enhanced by extracellular ATP activating P2X7R. Nat Med.
2010;16(12):1434-1438.

21. Jankovic D, Ganesan J, Bscheider M, et al. The Nlrp3 inflammasome regulates acute graft-versus-host disease. J Exp Med. 2013;210(10):1899-1910.

22. Eriguchi Y, Takashima S, Oka H, et al. Graft-versus-host disease disrupts intestinal microbial ecology by inhibiting Paneth cell production of alpha-defensins. Blood. 2012;120(1):223-231.

23. Simms-Waldrip TR, Sunkersett G, Coughlin LA, et al. Antibioticinduced depletion of anti-inflammatory Clostridia is associated with the development of graft-versus-host disease in pediatric stem cell transplantation patients. Biol Blood Marrow Transplant. 2017;23(5):820-829.

24. Jenq RR, Taur Y, Devlin SM, et al. Intestinal Blautia is associated with reduced death from graft-versus-host disease. Biol Blood Marrow Transplant. 2015;21(8):1373-1383

25. Mathewson ND, Jenq R, Mathew AV, et al. Gut microbiomederived metabolites modulate intestinal epithelial cell damage and mitigate graft-versus-host disease. Nat Immunol. 2016;17(5):505-513.

26. Wysocki CA, Burkett SB, Panoskaltsis-Mortari A, et al. Differential roles for CCR5 expression on donor T cells during graft-versus-host disease based on pretransplant conditioning. J Immunol. 2004;173(2):845-854.

27. Taylor PA, Ehrhardt MJ, Lees CJ, et al. Insights into the mechanism of FTY720 and compatibility with regulatory T cells for the inhibition of graft-versus-host disease (GVHD). Blood. 2007;110(9):3480-3488.

28. Kennedy GA, Varelias A, Vuckovic S, et al. Addition of interleukin-6 inhibition with tocilizumab to standard graftversus-host disease prophylaxis after allogeneic stem-cell transplantation: a phase 1/2 trial. Lancet Oncol. 2014;15(13):1451-1459.

29. Zeiser R, von Bubnoff N, Butler J, et al. Ruxolitinib for glucocorticoid-refractory acute graft-versus-host disease. $\mathrm{N}$ Engl J Med. 2020;382(19):1800-1810.

30. Choi SW, Braun T, Chang L, et al. Vorinostat plus tacrolimus and mycophenolate to prevent graft-versus-host disease after related-donor reduced-intensity conditioning allogeneic haemopoietic stem-cell transplantation: a phase $1 / 2$ trial. Lancet Oncol. 2014;15(1):87-95.

31. Joly AL, Deepti A, Seignez A, et al. The HSP90 inhibitor, 17AAG, protects the intestinal stem cell niche and inhibits graft versus host disease development. Oncogene. 2016;35(22):2842-2851.

32. Mukai S, Ogawa Y, Urano F, Kudo-Saito C, Kawakami Y, Tsubota K. Novel treatment of chronic graft-versus-host disease in mice using the ER stress reducer 4-phenylbutyric acid. Sci Rep. 2017;7:41939.

33. Tsalikis J, Pan Q, Tattoli I, et al. The transcriptional and splicing landscape of intestinal organoids undergoing nutrient starvation or endoplasmic reticulum stress. BMC Genomics. 2016;17(1):680.

34. Olivares S, Henkel AS. Hepatic Xbp1 gene deletion promotes endoplasmic reticulum stress-induced liver injury and apoptosis. J Biol Chem. 2015;290(50):30142-30151.

35. Niederreiter L, Fritz TM, Adolph TE, et al. ER stress transcription factor Xbp1 suppresses intestinal tumorigenesis and directs intestinal stem cells. J Exp Med. 2013;210(10):2041-2056.

36. Levine JE, Huber E, Hammer ST, et al. Low Paneth cell numbers at onset of gastrointestinal graft-versus-host disease identify patients at high risk for nonrelapse mortality. Blood. 2013;122(8):1505-1509.

37. Eriguchi Y, Nakamura K, Hashimoto D, et al. Decreased secretion 
of Paneth cell alpha-defensins in graft-versus-host disease. Transpl Infect Dis. 2015;17(5):702-706.

38. Holler E, Butzhammer P, Schmid K, et al. Metagenomic analysis of the stool microbiome in patients receiving allogeneic stem cell transplantation: loss of diversity is associated with use of systemic antibiotics and more pronounced in gastrointestinal graft-versus-host disease. Biol Blood Marrow Transplant. 2014;20(5):640-645.

39. Taur Y, Jenq RR, Perales MA, et al. The effects of intestinal tract bacterial diversity on mortality following allogeneic hematopoietic stem cell transplantation. Blood. 2014;124(7):1174-1182.

40. Hu P, Han Z, Couvillon AD, Kaufman RJ, Exton JH. Autocrine tumor necrosis factor alpha links endoplasmic reticulum stress to the membrane death receptor pathway through IRE1alphamediated NF-kappaB activation and down-regulation of TRAF2 expression. Mol Cell Biol. 2006;26(8):3071-3084.

41. Bettigole SE, Glimcher LH. Endoplasmic reticulum stress in immunity. Annu Rev Immunol. 2015;33;107-138.

42. Qiu Q, Zheng Z, Chang L, et al. Toll-like receptor-mediated IRE1alpha activation as a therapeutic target for inflammatory arthritis. EMBO J. 2013;32(18):2477-2490.

43. Denis RG, Arruda AP, Romanatto T, et al. TNF-alpha transiently induces endoplasmic reticulum stress and an incomplete unfolded protein response in the hypothalamus. Neuroscience. 2010;170(4):1035-1044.

44. Chan SMH, Lowe MP, Bernard A, Miller AA, Herbert TP. The inositol-requiring enzyme 1 (IRE1alpha) RNAse inhibitor, 4 micro8C, is also a potent cellular antioxidant. Biochem J. 2018;475(5):923-929.

45. Betts BC, Locke FL, Sagatys EM, et al. Inhibition of human dendritic cell ER stress response reduces T cell alloreactivity yet spares donor anti-tumor immunity. Front Immunol. 2018;9:2887.

46. Tang $\mathrm{CH}$, Ranatunga $\mathrm{S}$, Kriss $\mathrm{CL}$, et al. Inhibition of ER stressassociated IRE-1/XBP-1 pathway reduces leukemic cell survival. J Clin Invest. 2014;124(6):2585-2598.

47. Hassan IH, Zhang MS, Powers LS, et al. Influenza A viral replication is blocked by inhibition of the inositol-requiring enzyme 1 (IRE1) stress pathway. J Biol Chem. 2012;287(7):4679-4689.

48. Reimold AM, Iwakoshi NN, Manis J, et al. Plasma cell differentiation requires the transcription factor XBP-1. Nature. 2001;412(6844):300-307.

49. Schutt SD, Wu Y, Tang CH, et al. Inhibition of the IRE-1alpha/XBP1 pathway prevents chronic GVHD and preserves the GVL effect in mice. Blood Adv. 2018;2(4):414-427. 